\title{
Calibrating a global three-dimensional biogeochemical ocean model (MOPS-1.0)
}

\author{
Iris Kriest $^{1}$, Volkmar Sauerland ${ }^{2}$, Samar Khatiwala ${ }^{3}$, Anand Srivastav ${ }^{2}$, and Andreas Oschlies ${ }^{1}$ \\ ${ }^{1}$ GEOMAR Helmholtz-Zentrum für Ozeanforschung Kiel, Düsternbrooker Weg 20, 24105 Kiel, Germany \\ ${ }^{2}$ Institut für Informatik, Christian-Albrechts-Universität zu Kiel, Christian-Albrechts-Platz 4, 24098 Kiel, Germany \\ ${ }^{3}$ Department of Earth Sciences, University of Oxford, South Parks Road, Oxford OX1 3AN, UK
}

Correspondence to: Iris Kriest (ikriest@geomar.de)

Received: 4 July 2016 - Published in Geosci. Model Dev. Discuss.: 18 July 2016

Revised: 8 December 2016 - Accepted: 14 December 2016 - Published: 9 January 2017

\begin{abstract}
Global biogeochemical ocean models contain a variety of different biogeochemical components and often much simplified representations of complex dynamical interactions, which are described by many $(\approx 10$ to $\approx 100$ ) parameters. The values of many of these parameters are empirically difficult to constrain, due to the fact that in the models they represent processes for a range of different groups of organisms at the same time, while even for single species parameter values are often difficult to determine in situ. Therefore, these models are subject to a high level of parametric uncertainty. This may be of consequence for their skill with respect to accurately describing the relevant features of the present ocean, as well as their sensitivity to possible environmental changes.

We here present a framework for the calibration of global biogeochemical ocean models on short and long timescales. The framework combines an offline approach for transport of biogeochemical tracers with an estimation of distribution algorithm (Covariance Matrix Adaption Evolution Strategy, CMA-ES). We explore the performance and capability of this framework by five different optimizations of six biogeochemical parameters of a global biogeochemical model, simulated over 3000 years. First, a twin experiment explores the feasibility of this approach. Four optimizations against a climatology of observations of annual mean dissolved nutrients and oxygen determine the extent to which different setups of the optimization influence model fit and parameter estimates. Because the misfit function applied focuses on the large-scale distribution of inorganic biogeochemical tracers, parameters that act on large spatial and temporal scales are determined earliest, and with the least spread. Parameters
\end{abstract}

more closely tied to surface biology, which act on shorter timescales, are more difficult to determine. In particular, the search for optimum zooplankton parameters can benefit from a sound knowledge of maximum and minimum parameter values, leading to a more efficient optimization. It is encouraging that, although the misfit function does not contain any direct information about biogeochemical turnover, the optimized models nevertheless provide a better fit to observed global biogeochemical fluxes.

\section{Introduction}

Global ocean models that simulate biogeochemical interactions are subject to many uncertainties, among them those related to initial conditions, forcing, and parameterizations of physical and biological processes, as well as the adequacy of the chosen model complexity with respect to the scientific problem under investigation. It is generally assumed that all these "input" factors affect the simulation results in ways that may be different for different models, but a thorough understanding of how uncertainties in input map onto model output (residuals, i.e., deviations from the true state) is still lacking. Quantitative estimates of the effect of model uncertainty on model residuals are generally obtained from individual sensitivity studies and model intercomparison or model ensemble studies, where the spread of model results is regarded as a measure of model uncertainty. This procedure is, for example, followed in the assessment reports of the Intergovernmental Project of Climate Change (IPCC). The Ocean Carbon Model Intercomparison Project (OCMIP) applied a strict 
protocol regarding the description of biogeochemical processes to a suite of different ocean circulation models to show that the effect of uncertainties in the simulated circulation on biogeochemical tracer distributions and their residuals can be considerable (Orr et al., 2001; Najjar et al., 2007). However, the effect of uncertainties in the formulation of biogeochemical models on simulated biogeochemical tracers and fluxes can be of similar magnitude (Kriest et al., 2010) and is often difficult to disentangle from other sources of uncertainty (e.g., Cabre et al., 2015; Seferian et al., 2016). One reason for diverging results of global biogeochemical models can be related to the uncertainty with respect to biological constants and equations. In addition to often poorly constrained parameters, it is, so far, not even clear how complex a biogeochemical model should be (e.g., what state variables it should contain) in order to realistically reproduce observed global tracer distributions (Kriest et al., 2012). As a consequence, the diversity of biogeochemical models ranges from simple, "nutrient-only" models to far more complex ones, comprising different elemental cycles and biological components.

Uncertainties in biogeochemical model setup partly arise from sparse observations, particularly in the open ocean and during winter season in the high latitudes (Kriest et al., 2010). Further, the combined effects of shallow and deep biogeochemistry and ocean circulation introduce a variety of timescales, from minutes to millennia, hampering a complete and thorough investigation of the combined effects of the different process parameterizations. Finally, even quite simple biogeochemical models are often characterized by nonlinear interactions, complicating the a posteriori analysis of model results. By performing a relatively "coarse sweep" of the multidimensional model parameter space, Kriest et al. (2010, 2012) illustrated the impact of different model complexities and parameter sets on simulated tracers and their fit to observations. This first attempt to systematically explore the impacts of biogeochemical parameter uncertainty in global models may well have missed optimal regions in parameter space, making it difficult to decide whether a model performs badly due to ill-chosen parameters, or due to an inappropriate model structure. The development of automatic optimization of global ocean biogeochemical models that is the goal of this study should enable a more thorough search for "best" parameters, and thus facilitate inter-model comparison.

An under-sampled ocean, together with a large variety of timescales and space scales and a high level of structural model complexity, poses a challenge for optimization, and for a full, and dense enough, scan of the parameter space on a global scale. Therefore, optimization of marine biogeochemical models has mostly been carried out in a local, zeroor one-dimensional setting (e.g., Fasham and Evans, 1995; Athias et al., 2000; Rückelt et al., 2010; Ward et al., 2010). The variability of biogeochemical processes has been addressed by simultaneous optimization at different sites (and physical forcings) in the North Atlantic by Schartau and Oschlies (2003a, b). Given the high computational demands, and the sparsity of biogeochemical data on a global scale, attempts to address the indeterminacy of global simulations of ocean biogeochemistry via optimization have resorted to rather simple biogeochemical systems (Kwon and Primeau, 2006,2008 ) or to rather coarse physical model resolution (Tjiputra et al., 2007). To constrain parameters related to dissolved organic matter production and decay on short and long timescales, Letscher et al. (2015) alternated between a simplified biogeochemical system and a more complex model, which is limited in terms of spinup time. Recent attempts have begun to combine complex, local models and a detailed three-dimensional global environment for optimization (Hemmings et al., 2015). To our knowledge, however, the experiments presented here are the first ones that, for a stateof-the-art global biogeochemical ocean model, carry out a parameter optimization that targets parameters relevant for biogeochemical processes on both large and small scales in the full spatio-temporal domain.

In this paper we first test the global biogeochemical model optimization against synthetic data, derived from a previous model experiment with perturbed model parameters in socalled twin experiments. We then present four optimizations against a global, synoptic data set of observed phosphate, nitrate, and oxygen.

\section{Methods}

\subsection{Biogeochemical ocean model}

\subsubsection{Circulation framework}

For easy and generic coupling between different biogeochemical models and circulation fields, as well as fast and efficient computation, we use the Transport Matrix Method (TMM), developed by Samar Khatiwala (Khatiwala, 2007), and available via Github (https://github.com/ samarkhatiwala/tmm). This efficient "offline" method for ocean passive tracer transport represents advection and mixing in the form of transport matrices that have been calculated from an ocean circulation model simulation prior to the biogeochemical simulations performed here.

For optimization, we use the TMM with monthly mean transport matrices derived from a $2.8^{\circ}$ global configuration of the MIT ocean model with 15 levels in the vertical (Marshall et al., 1997). Using this rather coarse spatial grid, a time step length of $1 / 2$ day for tracer transport and $1 / 16$ day for biogeochemical interactions, each biogeochemical model setup with seven tracers has been simulated for 3000 years, after which most of the tracers approach steady state.

\subsubsection{Biogeochemical model}

The biogeochemical model employed as representative of current state-of-the-art models is the same as presented by Kriest and Oschlies (2015, hereafter called MOPS - 
Model of Oceanic Pelagic Stoichiometry), and we only describe it briefly here. Based on phosphorus, it consists of seven tracers, namely phosphate, dissolved inorganic nitrogen (hereafter termed and compared to nitrate), phytoplankton, zooplankton, detritus, dissolved organic matter (DOM), and oxygen. For conversion between the different elements we apply a constant global stoichiometry of $R_{-\mathrm{O}_{2}: \mathrm{P}}=170 \mathrm{mmol} \mathrm{O}_{2}: \mathrm{mmol} \mathrm{P}$ for the ratio between $\mathrm{O}_{2}: \mathrm{P}$, and $16 \mathrm{mmol} \mathrm{N}: \mathrm{mmol} \mathrm{P}$ for the $\mathrm{N}: \mathrm{P}$ ratio of particular and dissolved organic matter. The stoichiometry of aerobic and anaerobic remineralization is based on Paulmier et al. (2009). Remineralization of detritus and DOM is parameterized via a constant nominal remineralization rate, $r=0.05$ $\left(\mathrm{d}^{-1}\right)$. However, aerobic remineralization is restricted to regions with sufficient oxygen. If oxygen declines, nitrate is used as electron acceptor, thereby mimicking denitrification. If both oxygen and nitrate are depleted, remineralization of organic matter is suppressed in the model. Aerobic and anaerobic remineralization are parameterized as saturation (Monod-type) curves that regulate the rates of these processes using either oxidant, as well as the inhibition of denitrification by oxygen. Thus, the actual remineralization rate may differ from $r$, depending on oxidant availability. Temperature dependent nitrogen fixation resupplies fixed nitrogen lost through denitrification via relaxation at the sea surface to the stoichiometric ratio of 16 . Thus, while the total phosphate inventory is conserved, the oxygen and fixed nitrogen inventory may change during the course of the simulation, with the long-term, steady-state inventory depending on physics and biogeochemistry (Kriest and Oschlies, 2015).

Sinking of detritus is simulated using a sinking speed increasing with depth $w=a z\left(\mathrm{~d}^{-1}\right)$. Assuming a constant remineralization rate $r$, equilibrium conditions, and the absence of horizontal or vertical advection, this would result in a particle flux profile defined by $F(z) \propto z^{-b}$, where $b=r / a$ (see also Kriest and Oschlies, 2008). For better comparison to observed particle flux profiles (e.g., Martin et al., 1987), in the following we express the sinking speed via the parameter $b=r / a$ (see Kriest and Oschlies, 2008). The model also includes burial of particulate organic phosphorus and nitrogen arriving at the sea floor, which is resupplied globally as phosphate and nitrate via river runoff (Kriest and Oschlies, 2013).

Simulating both surface (primary production, grazing, egestion and excretion by zooplankton) as well as deep (sinking and decay of organic matter) processes before the background of ocean circulation and seasonally varying forcing, the model thus encompasses processes that act on a variety of timescales, from the order of hours to days (surface) to months and years.

\subsection{The CMA-ES optimization algorithm}

\subsubsection{Population based search heuristics}

The TMM as described above is fast enough to be used together with meta-heuristic methods for parameter optimization, such as Evolutionary Algorithms (EAs) or Estimation of Distribution Algorithms (EDAs). Although these methods require more function evaluations to converge to some local optimum than gradient based methods, they are of advantage in complicated, irregular "search landscapes" with local optima (which might be far worse than the global optimum), or discontinuities.

The common goal of such population based metaheuristics is to strike a good balance of both search properties, exploration (search for promising solutions in a wide area of the search space), and exploitation (search within small regions around good solutions to quickly reach local optima). Classical evolutionary algorithms as depicted on the left of Fig. 1 mimic principles of natural evolution to pursue that goal. They use randomized procedures to select, combine, mutate, and reinsert candidate solutions (individuals) from/into a given solution set (population). In each iteration, these mechanisms (red operations in Fig. 1) indirectly imply a probability distribution on the search space with respect to which individuals are likely to appear in the next "generation". The implied probability distribution changes in each generation, tending to increase the probabilities of good solutions and to decrease the probabilities of poor solutions due to the survival-of-the-fittest principle.

In contrast to classical EAs, Estimation of Distribution Algorithms (sketched on the right of Fig. 1) use an explicit (parameterized) probability distribution from which candidate solutions are sampled directly. In each iteration, the probability distribution is also updated directly by utilizing good solutions of the current iteration. Good solutions of preceding iterations are (optionally) considered by involving preceding probability distributions into the update process using auxiliary variables. Evolutionary frameworks use operators (EAs) and probability distributions (EDAs) that are appropriate for the search space under consideration. For example, so-called quantum inspired evolutionary algorithms (QiEA) have been shown to be very suitable EDAs for binary problems (e.g., Kliemann et al., 2013; Patvardhan et al., 2015, 2016). QiEA versions for continuous problems have also been investigated in the literature (Babu et al., 2009).

Here we use a state-of-the-art EDA for optimization of (firstly) six parameters. Our task can be classified as a continuous optimization problem with bound constraints, i.e., boundaries for the parameters. One appropriate EA/EDA tool is the Covariance Matrix Adaption Evolution Strategy (CMA-ES; Hansen and Ostermeier, 2001; Hansen, 2006), which has shown good performance with respect to quality and efficiency (in terms of function evaluations) in similar applications. Hansen et al. (2010) compare 31 algorithms on 
Evolutionary algorithm (EA)

(a)

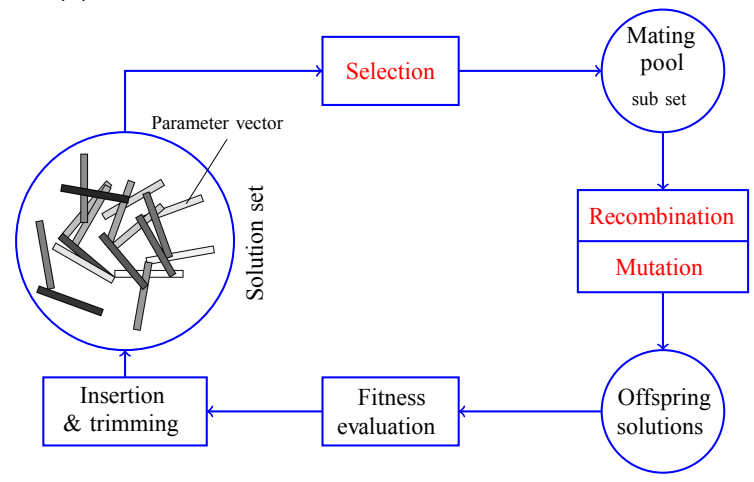

Estimation of distribution algorithm (EDA)

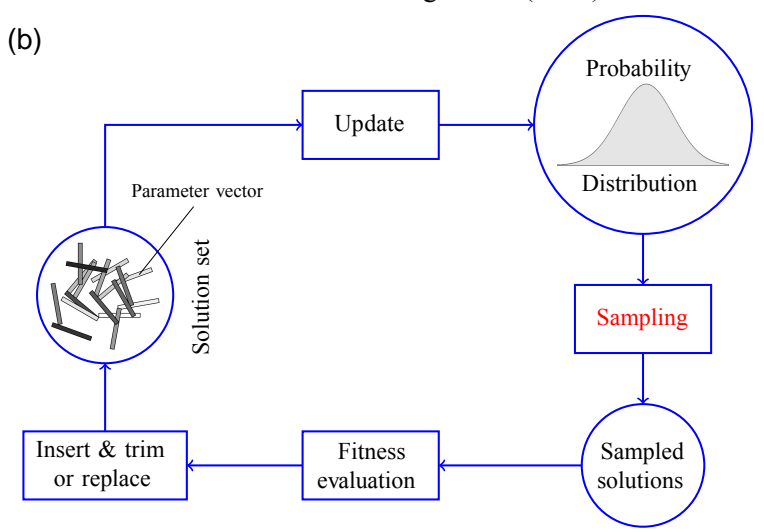

Figure 1. A general EA (left) and EDA (right) schematic. Cycles represent sets of solutions (vectors of BGC parameters in our case) or an explicit probability distribution from which new solutions can be drawn. Rectangle symbols depict operations. Operations displayed in red font depend on random decisions. EA: a set of candidate solutions (population) is iteratively updated. In each generation, candidate solutions compete to form a mating pool which is realized by a random selection operator. Offspring solutions are produced by recombining mates and/or introducing some mutation. Finally, there is a fitness based insertion back into the population, which is usually trimmed to a predefined population size. The random operators selection, recombination, and mutation imply an implicit probability distribution on the search space with respect to which solutions are likely to appear in the next generation. EDA: candidate solutions of the current iteration's population are used to update an explicit probability distribution such that the likelihood to sample good solutions increases. New candidate solutions are directly sampled from the probability distribution. Usually, the realization of the probability distribution update ensures that information of former solutions fades out slowly, resisting for several iterations. Therefore, the population may be smaller as an EA population and even be replaced with the entire set of new samples, which is the case for the CMA-ES algorithm we use.

a test bed of 24 continuous benchmark functions presented in Hansen et al. (2009a), finding CMA-ES versions to perform well, particularly on multi-modal test functions. CMAES is invariant regarding both order-preserving transformations of the objective function and rotations and translations of the search space. Invariances of a strategy justify generalizations of empirical results, which encouraged us to choose CMA-ES for our application.

We essentially follow the description of the $\left(\mu / \mu_{\mathrm{w}}, \lambda\right)-$ CMA-ES in Hansen (2016). In Sect. 2.2.2, we illustrate how the distribution is sampled and modified. For the sake of completeness, we present the guiding ideas behind the exact procedures in Sect. 2.2.3-2.2.6. The algorithm outline can be found in Sect. 2.3. This basic version does not consider bound constraints. We therefore use a penalty function based boundary handling (Hansen et al., 2009b) which we will briefly explain in Sect. 2.2.7.

\subsubsection{Normal distributions}

In CMA-ES the distribution from which candidate solutions (BGC parameter vectors in our application) are sampled is a multi-variate normal distribution. It generalizes the usual normal distribution, also known as Gaussian distribution, from $\mathbb{R}$ to the vector space $\mathbb{R}^{n}$ with arbitrary dimension $n$, given by the number of biogeochemical parameters to be estimated. The position and the shape of the one-dimensional normal distribution (more precisely, its density function) is uniquely defined by its mean and its variance, respectively.

A measure of the "diversity" of a probability distribution is the so-called (differential) entropy. For a given variance, the normal distribution has the maximum entropy amongst all distributions with the same variance (Cover and Thomas, 2006; Hansen, 2016). Entropy is used as an index of diversity, though it does not directly mean the same as diversity (Jost, 2006).

An EDA that works with Gaussian distributions is supposed to carefully update both defining distribution parameters mean and variance, in order to balance its exploration and exploitation ability. This update process is illustrated in Fig. 2. The left side shows a run of the CMA-ES algorithm on a uni-variate test function (a misuse to some degree, as CMA-ES is actually not suggested to be applied with problem dimensions less than 5). The test function has many local optima in which a gradient based search might get stuck. From the distributions (the blue density functions), we draw 10 samples per iteration (some samples more than the suggested default number, which depends on the problem dimension; cf. Sect. 2.3.1). Each sample together with its function value is marked with a dot. The distribution is updated by involving the better half (CMA-ES default portion) of the samples (blue dots). Drawing more samples per iteration generally improves the exploration capability of the algorithm but requires correspondingly more function evaluations. We can observe that the mean of the distribution is 

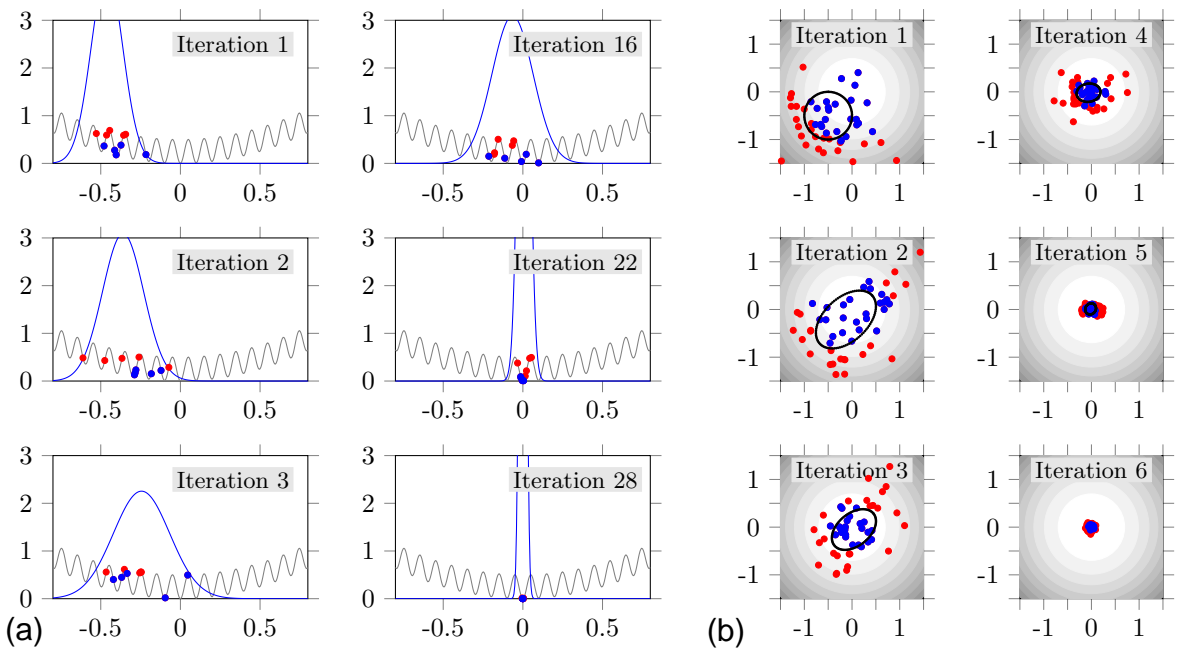

Figure 2. Iterations of the CMA-ES applied to test functions. Left: a uni-variate Griewank-type function $f$ (grey curve). In each iteration we draw $\lambda=10$ samples from the normal distribution (blue curve). For each sample $x_{i}$, the pair $\left(x_{i}, f\left(x_{i}\right)\right)$ is marked with a dot. The $\mu=\frac{\lambda}{2}=5$ better samples (blue dots) are involved in the normal distribution update for the next iteration. Right: two-dimensional sphere function. Here, samples are marked with dots, while function values are indicated by the grey levels in the counter plots; the $i$ th grey level represents the range $\left[\frac{i-1}{2}, \frac{i}{2}\right.$. More samples (50) than necessary are used to update the distribution, which is indicated by its standard deviation ellipse (black), here. Distributions tend to elongate into directions of descent (iteration 2). For the convex example function, the algorithm converges after a few iterations.

attracted towards the good samples, then. Also, the distribution shape widens, after good samples had some distance to each other and/or some distance to the current mean. Vice versa, if all good samples are close to the mean, the shape will narrow, again. Now, the mean of the distribution is supposed to drift towards the global optimum and should then start to narrow more and more. This behavior is observed in iterations 16,22 , and 28 . So, when necessary, the procedure is supposed to become less exploring but more exploiting.

Similarly to the definition of the uni-variate Gaussian distribution by mean and variance, a multi-variate normal distribution can be uniquely identified by a mean vector $\overline{\boldsymbol{x}}$ and a positive definite matrix $\mathbf{C}$ of covariances, respectively, and is denoted by $\mathcal{N}(\overline{\boldsymbol{x}}, \mathbf{C})$. Again, the mean defines the center of the distribution, while the covariance matrix defines its shape. The area of 1 standard deviation which is an interval $[x-\sigma, x+\sigma]$ in the one-dimensional case becomes an $n$ dimensional ellipsoid, now (cf. the ellipses on the right side of Fig. 2 for $n=2$ ). It can be shown that the principal axes of the ellipsoid correspond to C's eigenvalues and eigenvectors, respectively. More precisely, an eigenvector defines the orientation of a principal axis and the square root of the corresponding eigenvalue defines the length of that principal axis.

\subsubsection{Sampling the distribution}

Sampling a multi-variate normal distribution $\mathcal{N}(\overline{\boldsymbol{x}}, \mathbf{C})$ can be practically implemented using an eigendecomposition $\mathbf{C}=$ $\mathbf{B D}^{2} \mathbf{B}^{\mathrm{T}}$, where $\mathbf{D}^{2}$ is a diagonal matrix of eigenvalues of $\mathbf{C}$ and $\mathbf{B}$ is a matrix of corresponding orthonormal eigenvec- tors of $\mathbf{C}$. One sample $\boldsymbol{x} \in \mathbb{R}^{n}$ of $\mathcal{N}(\overline{\boldsymbol{x}}, \mathbf{C})$ can be realized by drawing $n$ independent random numbers from the uni-variate standard normal distribution $\mathcal{N}(0,1)$ to be the components of a random vector $\boldsymbol{z} \in \mathbb{R}^{n}$ and setting $\boldsymbol{x}=\overline{\boldsymbol{x}}+\mathbf{B D} \boldsymbol{z}$.

Note that for our problem there are bound constraints on the parameters such that samples of a normal distribution might be infeasible, regardless of whether the distribution mean is feasible or not. However, a boundary handling procedure (see Sect. 2.2.7) will ensure that the optimization result of CMA-ES is feasible.

\subsubsection{Updating the distribution: basic principle}

Empirical (re)estimates $\overline{\boldsymbol{x}}_{\text {emp }}$ and $\mathbf{C}_{\text {emp }}$ of the distribution parameters can be calculated from a set $S=\left\{\boldsymbol{x}_{1}, \ldots, \boldsymbol{x}_{\lambda}\right\}$ of $\lambda$ samples, such that the expectation of $\overline{\boldsymbol{x}}_{\mathrm{emp}}$ is $\overline{\boldsymbol{x}}$ and the expectation of $\mathbf{C}_{\mathrm{emp}}$ is $\mathbf{C}$ :

$$
\begin{aligned}
\overline{\boldsymbol{x}}_{\mathrm{emp}} & =\frac{1}{\lambda} \sum_{i=1}^{\lambda} \boldsymbol{x}_{i}, \\
\mathbf{C}_{\mathrm{emp}} & =\frac{1}{\lambda-1} \sum_{i=1}^{\lambda}\left(\boldsymbol{x}_{i}-\overline{\boldsymbol{x}}_{\mathrm{emp}}\right)\left(\boldsymbol{x}_{i}-\overline{\boldsymbol{x}}_{\mathrm{emp}}\right)^{\mathrm{T}} .
\end{aligned}
$$

Note that each vector $v$ in this work is a column vector and its transposed vector $\boldsymbol{v}^{\mathrm{T}}$ is a row vector. The products under the sum in the second formula are therefore $n$-by- $n$ matrices.

Clearly, the estimates become more reliable the larger $\lambda$ is. We may assume that the population $S$ is increasingly ordered (ranked) with respect to the considered objective 
Table 1. Operational constants of the CMA-ES algorithm (cf. the initialization in Algorithm 1).

\begin{tabular}{lll}
\hline Selection and recombination & Step size control & Covariance matrix adaption \\
\hline$\lambda=4+\lfloor 3 \log n\rfloor$ & $\chi=\sqrt{n}\left(1-\frac{1}{4 n}+\frac{1}{21 n^{2}}\right)$ & $c_{\mathrm{c}}=\frac{4+\mu_{\mathrm{eff}} / n}{n+4+2 \mu_{\mathrm{eff}} / n}$ \\
$\mu=\left\lfloor\frac{\lambda}{2}\right\rfloor$ & $c_{\sigma}=\frac{\mu_{\mathrm{eff}}+2}{n+\mu_{\mathrm{eff}}+5}$ & $\operatorname{cin}\left(1-c 1,2 \frac{\mu_{\mathrm{eff}}+1 / \mu_{\mathrm{eff}}-2}{(n+2)^{2}+\mu_{\mathrm{eff}}}\right)$ \\
$w_{i}=\frac{\log (\mu+0.5)-\log (i)}{\sum_{j=1}^{\mu} \log (\mu+0.5)-\log (j)}$ & & \\
$\mu_{\mathrm{eff}}=\frac{\left(\sum_{i=1}^{\mu} w_{i}\right)^{2}}{\sum_{i=1}^{\mu} w_{i}^{2}}=\frac{1}{\sum_{i=1}^{\mu} w_{i}^{2}}$ & & \\
\hline
\end{tabular}

function $f: \mathbb{R}^{n} \longrightarrow \mathbb{R}$, that is, $f\left(\boldsymbol{x}_{1}\right) \leq f\left(\boldsymbol{x}_{2}\right) \ldots \leq f\left(\boldsymbol{x}_{\lambda}\right)$. Now, by involving only the better half of $\mu=\left\lfloor\frac{\lambda}{2}\right\rfloor$ samples, their distribution estimate $\mathcal{N}\left(\overline{\boldsymbol{x}}_{\mu}, \mathbf{C}_{\mu}\right)$ with corresponding parameters $\overline{\boldsymbol{x}}_{\mu}$ and $\mathbf{C}_{\mu}$ will be biased towards reproducing $\mu$ samples with higher probability than the other $\lambda-\mu$ samples. CMA-ES uses positive values $w_{1} \geq w_{2} \geq \ldots \geq w_{\mu}$ with $\sum_{i=1}^{n} w_{i}=1$ to give solutions a rank dependent weight in the updating process of both, $\overline{\boldsymbol{x}}_{\mu}$ and $\mathbf{C}_{\mu}$. The exact CMAES formula for the $w$ values and information about its background is found in Sect. 2.3.1. The new mean is, thus, calculated as $\overline{\boldsymbol{x}}_{\mu}=\sum_{i=1}^{\mu} w_{i} \boldsymbol{x}_{i}$. A subtlety is the choice of the reference mean value used for estimating $\mathbf{C}_{\mu}$. Instead of the new empirical mean $\overline{\boldsymbol{x}}_{\mu}$, the mean $\overline{\boldsymbol{x}}$ of the former distribution is chosen and yields

$\mathbf{C}_{\mu}=\sum_{i=1}^{\mu} w_{i}\left(\boldsymbol{x}_{i}-\overline{\boldsymbol{x}}\right)\left(\boldsymbol{x}_{i}-\overline{\boldsymbol{x}}\right)^{\mathrm{T}}$

It has the effect that the new distribution is elongated into directions of descent (see iteration 2 in the right example of Fig. 2).

\subsubsection{Updating the distribution: reliability with small populations}

As mentioned above, reliable distribution estimates require a sufficiently large number of samples. However, for a competitive computational performance we must get along with a rather small number of samples. CMA-ES therefore involves the information of former populations by updating the covariance matrix $\mathbf{C}$ to be a (convex) combination of both the current $\mathbf{C}$ and its estimate $\mathbf{C}_{\mu}$, that is,

$\mathbf{C} \leftarrow\left(1-c_{\mu}\right) \mathbf{C}+c_{\mu} \mathbf{C}_{\mu}$.

Using this formula, it can be shown that $37 \%$ of the current matrix C's information dates back at least $\left\lfloor\frac{1}{c_{\mu}}\right\rfloor$ generations; that is, the choice of the smoothing factor $c_{\mu}$ decides the backward time horizon of the update procedure. The smaller the factor $c_{\mu}$ in Eq. (2) is, the more former samples contribute to the current distribution estimate, slowing down learning but being more reliable, with fewer samples per iteration. For example, the experiments in this paper use $n=6$ parameters and $\lambda=10$ samples per generation. Using Eq. (2) to update $C$ and the (compromise) $c_{\mu}$ value defined for CMAES (see Table 1), the samples of the last 23 iterations would contribute roughly $63 \%$ of the overall information in $C$.

Another feature that facilitates small population sizes $\lambda$ is to calculate and update a vector $\boldsymbol{p}_{\mathrm{c}}$ that represents iterationaveraged changes of the distribution mean and to use $\boldsymbol{p}_{\mathrm{c}}$ for a so-called rank-one estimate $\mathbf{C}_{1}=\boldsymbol{p}_{\mathrm{c}} \boldsymbol{p}_{\mathrm{c}}^{\mathrm{T}}$ of the covariance matrix. The idea behind this approach is that, using $\mathbf{C}_{\mu}$, distribution elongations into directions of descent do not distinguish for the sign of the directions. The use of the vector $\boldsymbol{p}_{\mathrm{c}}$ (called the evolution path) mitigates this effect. Consecutive changes of the distribution mean into opposite directions would cancel out each other. Similar to the smoothing with factor $c_{\mu}$ in the update of $\mathbf{C}$, above, the update of $\boldsymbol{p}_{\mathrm{c}}$ is done with a smoothing factor $c_{\mathrm{c}}$. With a further smoothing factor $c_{1}$ for the rank-one estimate $\mathbf{C}_{1}$, the combined covariance matrix update reads

$\mathbf{C} \leftarrow\left(1-c_{\mu}-c_{1}\right) \mathbf{C}+c_{\mu} \mathbf{C}_{\mu}+c_{1} \mathbf{C}_{1}$.

While $\mathbf{C}_{\mu}$ efficiently involves information from the current population in the update process, $\mathbf{C}_{1}$ exploits correlations between generations. The former is important in large populations; the latter is particularly important in small populations.

\subsubsection{Step size control}

Finally, there is an additional explicit adaption of the overall scale (the step size) of the distribution by adapting a scaling factor $\sigma$, actually using $\mathcal{N}\left(\overline{\boldsymbol{x}}, \sigma^{2} \mathbf{C}\right)$ instead of $\mathcal{N}(\overline{\boldsymbol{x}}, \mathbf{C})$. Similar to the evolution path $\boldsymbol{p}_{\mathrm{c}}$ for the rank-one covariance matrix estimates above, the adaption of the scale $\sigma$ involves an evolution path $\boldsymbol{p}_{\sigma}$ that mirrors cumulative changes in the mean. The difference between the update formulas of both evolution paths $\boldsymbol{p}_{\sigma}$ and $\boldsymbol{p}_{\mathrm{c}}$ is that for $\boldsymbol{p}_{\sigma}$ each change is re-scaled (normalized) with respect to the isotropic normal distribution $\mathcal{N}(0, \mathbf{I})$. Since covariances are always reestimated with respect to the mean of the former iteration (cf. Eq. 1), the expected normalized change of the distribution mean per iteration is therefore the expected length of a sample of $\mathcal{N}(0, \mathbf{I})$, which is

$\chi:=\mathbb{E}(\|\mathcal{N}(0, \mathbf{I})\|) \approx \sqrt{n}\left(1-\frac{1}{4 n}+\frac{1}{21 n^{2}}\right)$. 
Now, a rather small length $\left\|\boldsymbol{p}_{\sigma}\right\|$ compared to $\chi$ indicates that consecutive normalized moves of the mean canceled each other out, meaning that the overall scale of the distribution should be reduced with $\sigma$. Vice versa, an evolution path $\boldsymbol{p}_{\sigma}$ longer than $\chi$ indicates consecutive distribution drifts into correlated directions, which justifies a larger overall scale of the distribution.

\subsubsection{Boundary handling}

In order to consider boundary constraints, we use the procedure proposed in Hansen et al. (2009b, Sect. IV B) for CMA-ES. It applies if the distribution mean runs out of bounds. In this case, the fitness of an unfeasible sample $\boldsymbol{x}$ becomes the sum of the fitness of its closest feasible point $\boldsymbol{x}_{\text {feas }}$ and a weighted quadratic penalty function of its distance $\left\|\boldsymbol{x}-\boldsymbol{x}_{\text {feas }}\right\|$ to the feasible box (to $\boldsymbol{x}_{\text {feas }}$ ). Feasible samples are not penalized; i.e., the penalty function is 0 within the feasible box. Thus, the minimum of the sum of the actual fitness function and the penalty function is taken inside the feasible box or on its boundary. The quadratic penalty function has coordinate-wise weights $\frac{\gamma_{i}}{\xi_{i}}$, where $\xi_{i}$ scales the outof-bounds distance in the $i$ th coordinate with regard to the shape of the current distribution. The $\gamma_{i}$ are suitably initialized with the range of former (unpenalized) objective function values and is multiplied by a constant $>1$ in every iteration in which $\bar{x}_{i}$ is more than 3 standard deviations off its bounds.

In our implementation of CMA-ES, the feasible box we operate on is the unit cube $[0,1]^{n} \subseteq \mathbb{R}^{n}$. The samples are then linearly transformed (encoded) with respect to the actual bound constraints before evaluating the objective (misfit) function.

\subsection{Implementation of the optimization algorithm}

\subsubsection{Algorithm outline}

The CMA-ES approach described in Sect. 2.2 allows for reliable covariance matrix estimates with a relatively small population size. The default population size of $\lambda=4+3 \log (n)$ individuals and all further operational constants are successively derived from the problem dimension $n$ as outlined in Table 1.

Here, $\mu$ counts the good portion of individuals that are selected from the $\lambda$ samples in each iteration and used to update the probability distribution. As mentioned in Sect. 2.2.4, sampled individuals are always sorted with respect to their function values $\left(f\left(\boldsymbol{x}_{\mathbf{1}}\right) \leq \ldots \leq f\left(\boldsymbol{x}_{\lambda}\right)\right)$.

The $\mu$ recombination weights $w_{i}$ sum up to 1 and are monotonically decreasing in order to give better selected samples a higher weight in the updating formulas. Hansen (2016) suggests using the value $\mu_{\text {eff }}$ as a quality measure for the weights and states that

$\mu_{\mathrm{eff}}=\frac{\lambda}{4}$

indicates a good choice. Indeed, Eq. (3) is approximately satisfied by the given weighting scheme. We can only briefly sketch the history behind the suggestion: with equal weights $\frac{1}{\mu}$ in the distribution update, all the best $\mu$ independent samples would count with the same influence. For this case it has been shown with an exemplary uni-modal function (the infinite-dimensional sphere function) that the setting $\mu=$ $0.27 \cdot \lambda$ is optimal in the sense that the "expected progress per sample" towards the global optimum is maximized (Hansen et al., 2015, Sect. 4.2.2) (cf. Beyer, 2001, Chap. 3.1.1 and 3.2.1.2). Hansen considers the value $\mu_{\text {eff }}$ to be a generalization of the number of selected independent samples that influence the distribution, consequently using the similar Eq. (3) for the case of rank dependent weights. Note that $\mu_{\mathrm{eff}}$ takes its maximum $\mu$ with equal weights and its minimum 1 if all but one weight are zero. Actually, theoretically optimal non-equal weights and, thus, the optimal value for $\mu_{\text {eff }}$, are also known for the infinite-dimensional sphere function (Arnold, 2006, Sect. 3.2). These include non-zero weights for all $\lambda$ samples and negative weights for the worse $\frac{\lambda}{2}$ samples (hence doubling the value of $\mu_{\text {eff }}$ ). However, negative weights are not considered to be a robust enough practical choice.

Together with the problem dimension $n$, the generalized number of independent selected samples $\mu_{\text {eff }}$ appears in the calculation of the four smoothing constants $c_{\sigma}, c_{\mathrm{c}}, c_{\mu}, c_{1}$ used in the update formulas of both the evolution paths and the covariance matrix. Their dependence on $n$ and $\mu_{\text {eff }}$ has been derived empirically. The constant $\chi$ (cf. Sect. 2.2.6) is approximately the expected norm of the $n$-dimensional standard normal distribution $\mathcal{N}(0, \mathbf{I})$.

The algorithm details are summarized in Algorithm 1.

It starts with the identity matrix I for the covariances, that is, with an isotropic distribution. Assuming the optimum solution to reside within the unit cube $[0,1]^{n} \subseteq \mathbb{R}^{n}$, the mean $\overline{\boldsymbol{x}}$ and the overall scale $\sigma$ are initialized according to Hansen (2016). Actually, having bound constraints (cf. Sect. 2.2.7), we operate on the unit cube and shift and scale obtained samples into their real bounds before calculating their objective function values. New samples are drawn as described in Sect. 2.2.3. The $\boldsymbol{y}_{k}$ correspond to the $\boldsymbol{x}_{k}-\overline{\boldsymbol{x}}$ considered there, divided by the step size $\sigma$. The new $\overline{\boldsymbol{x}}$ is calculated according to $\overline{\boldsymbol{x}}_{\mu}$ in Sect. 2.2.4. Note that $\overline{\boldsymbol{y}}$ is the $\sigma$-adjusted move of the mean, while $\overline{\boldsymbol{y}}^{*}$ adjusts the move of the mean with respect to the (isotropic) standard normal distribution. The evolution paths which cumulate the drifts of the distribution mean (adjusted with regard to the overall scale and with regard to isotropy, respectively) are updated using the corresponding smoothing factors. Here, the factors before $\overline{\boldsymbol{y}}$ and $\overline{\boldsymbol{y}}^{*}$ act as normalization constants (Hansen, 2016). Finally, the overall step size and the covariances are updated as described 
in Sect. 2.2.6 and 2.2.5, respectively. For the given weights $w$ the factor $\frac{c_{\sigma}}{1+c_{\sigma}}$ in the update formula of $\sigma$ is equal to a more general formulation used, e.g., in the CMA-ES tutorial (Hansen, 2016). We stop either after the predefined number of iterations or if the current population shows a flat misfit distribution, i.e., if the fitness of the better $70 \%$ of the individuals deviates less than $\epsilon=10^{-5}$ from the very best one.

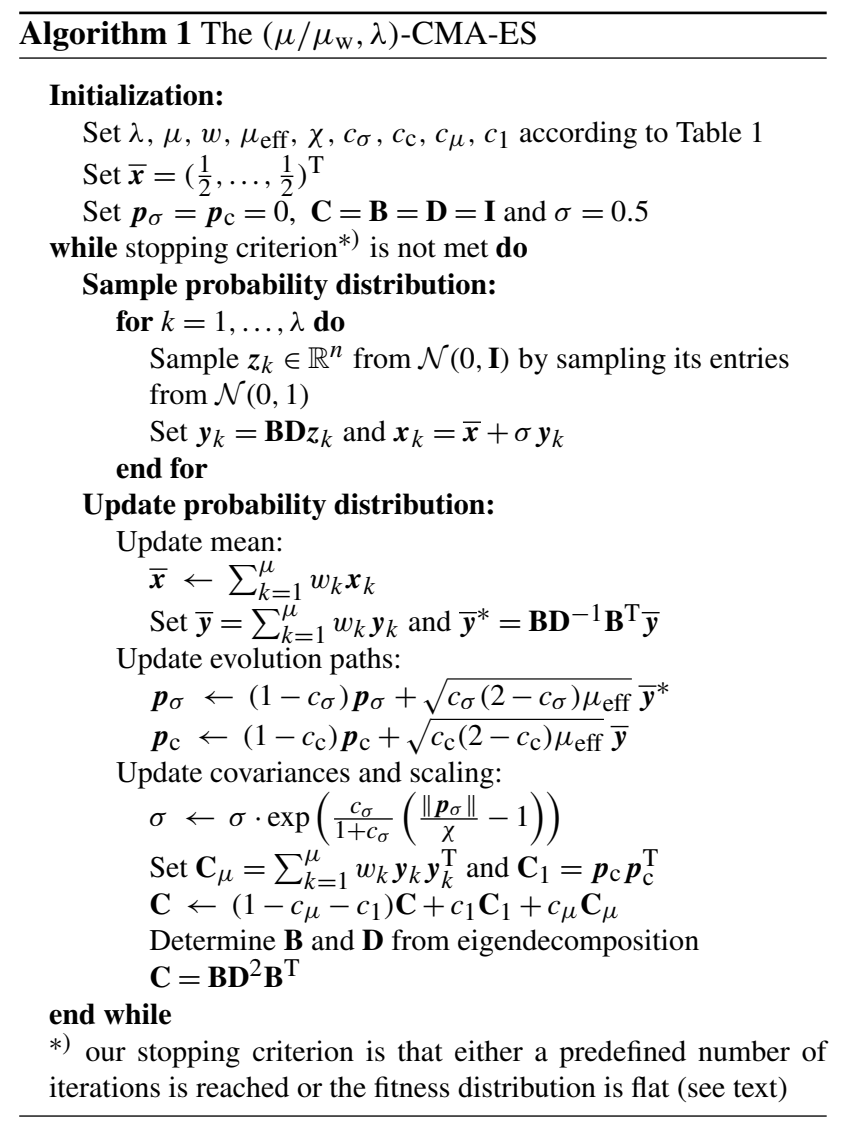

\subsubsection{Algorithm parallelization}

Our current technical implementation of the parallel framework can be easily transferred to other EAs/EDAs. The iterative optimization process is carried out via a series of chain jobs, where short serial jobs (the actual optimizer) that update the population of model evaluations ("individuals"; i.e., parameter sets for biogeochemistry) alternate with parallel jobs of function evaluations ("generations"), i.e., forward integrations of the coupled ocean model with different parameter sets. Parameters of the optimizer are population size $\lambda$ and the termination criterion for convergence; additionally, a maximum number of iterations.

As noted above, the framework presented here is set up such that a serial script serial. job calls the optimization routine (in our case CMA-ES), which computes a population of size $=\lambda$ of parameter vectors, stored in ASCII files. The same script then calls a parallel script paral lel . job, which starts $\lambda$ model simulations. During these simulations, the parameter files are read, and a spinup is carried out for each individual setup. The individual model runs then output the misfit function to specified files. When all jobs are finished, script parallel. job invokes script serial. job again, etc. Thus, communication between both alternating steps (creation of parameter vectors and computation of the resulting misfit function) is carried out by these parameter and misfit files. In addition, file nIter. txt keeps track of the progress of optimization, and provides the information on which generation is to be computed; it also contains the runtime parameters for the optimizer, CMA-ES. See the information in the Supplement for more details on how this setup works, and how to specify the biogeochemical and optimizer parameters used, e.g., in the work presented here.

\subsection{Misfit function}

As a first approach to optimization, we have calculated the root-mean-square error RMSE between simulated and observed (or twin) annual mean phosphate, nitrate, and oxygen concentrations on a global scale, weighted by the volume $V_{\mathrm{i}}$ of each individual grid box, expressed as a fraction of total ocean volume, $V_{\mathrm{T}}$. To sum the three different components of the misfit function, we have to divide them by some typical value. Here we use the global mean concentration of observed tracers. The resulting misfit function $J$ thus reads as

$J=\sum_{j=1}^{3} \frac{1}{\overline{o_{j}}} \sqrt{\sum_{i=1}^{N}\left(m_{i, j}-o_{i, j}\right)^{2} \frac{V_{i}}{V_{\mathrm{T}}}}$

for the annual mean concentrations of three tracers phosphate $(j=1)$, nitrate $(j=2)$, and oxygen $(j=3)$, at $N=52749$ locations (model grid boxes) of the model domain. $\overline{o_{j}}$ is the global average observed (or twin) concentration of the respective tracer. $m_{i, j}$ and $o_{i, j}$ are model and observations (or twin results), respectively. By weighting the model mismatch with volume, we put some emphasis on the deep ocean, down-weighting deviations in surface grid boxes relative to those of deep boxes. Thus, our misfit function serves more as a long timescale geochemical estimator, in contrast to a function that focuses on (rather fast) turnover in the surface layer.

\subsection{Parameters to be estimated}

Although the model contains more than 20 parameters (even more, if we consider the empirically derived parameters for benthic burial, nitrogen fixation, denitrification, and air-sea gas exchange; see Kriest and Oschlies, 2013, 2015), for this first approach we only consider six parameters for optimization. As a stringent test for the framework we chose parameters that encompass a large range of timescales and space scales, and reflect different trophic levels and dependencies between internal (interactions between compartments) and external (dependence on light) factors. We aimed to avoid si- 
Table 2. Experimental setup of optimization. "low" and "high" indicate boundary constraints of the optimizations, respectively.

\begin{tabular}{lrr|rr|rr|rr|rr|rr|r|r}
\hline \multirow{2}{*}{ Name } & \multicolumn{2}{c}{$R-\mathrm{O}_{2}: \mathrm{P}$} & \multicolumn{2}{c|}{$I_{\mathrm{c}}$} & \multicolumn{2}{c|}{$K_{\mathrm{PHY}}$} & \multicolumn{2}{c|}{$\mu_{\mathrm{ZOO}}$} & \multicolumn{2}{c|}{$\kappa_{\mathrm{ZOO}}$} & \multicolumn{2}{c}{$b^{*}$} \\
\cline { 2 - 12 } & low & high & low & high & low & high & low & high & low & high & low & high \\
\hline TWIN & 150 & 200 & 4.0 & 48 & 0.0001 & 0.5 & 0.1 & 4.0 & 0.0 & 10.0 & 0.4 & 1.8 \\
OBS-WIDE & 150 & 200 & 4.0 & 48 & 0.0001 & 0.5 & 0.1 & 4.0 & 0.0 & 10.0 & 0.4 & 1.8 \\
OBS-WIDE-20 & 150 & 200 & 4.0 & 48 & 0.0001 & 0.5 & 0.1 & 4.0 & 0.0 & 10.0 & 0.4 & 1.8 \\
OBS-NARR & 150 & 200 & 4.0 & 48 & 0.0001 & 0.5 & 1.0 & 3.0 & 1.6 & 4.8 & 0.4 & 1.8 \\
OBS-NARR-R & 150 & 200 & 4.0 & 48 & 0.0001 & 0.5 & 1.0 & 3.0 & 1.6 & 4.8 & 0.4 & 1.8 \\
\hline
\end{tabular}

* Note that from $b$ (the optimized parameter) in the model we calculate the rate of vertical increase in sinking speed $a$, always assuming a nominal detrital remineralization of $r=0.05 \mathrm{~d}^{-1}$. The resulting values for $a$ are 0.058275 (target (twin)), 0.125 (high), and 0.027778 (low)

multaneous optimization of parameters that are obviously related to each other, such as maximum growth rates and halfsaturation constants, or sinking speed and remineralization rate.

Four parameters are more relevant for biological interactions at the sea surface. Phytoplankton growth is controlled by the half-saturation for light $\left(I_{\mathrm{c}}\right.$, in $\left.\mathrm{W} \mathrm{m}^{-2}\right)$ and phosphate $\left(K_{\mathrm{PHY}}\right.$, in $\left.\mathrm{mmol} \mathrm{P} \mathrm{m}{ }^{-3}\right)$. For optimization of zooplankton parameters we chose its maximum grazing rate $\left(\mu_{\mathrm{ZOO}}\right.$, in $\left.\mathrm{d}^{-1}\right)$ and quadratic mortality rate $\left(\kappa \mathrm{ZOO}\right.$, in $\left.\left(\mathrm{mmol} \mathrm{P} \mathrm{m}^{-3}\right)^{-1} \mathrm{~d}^{-1}\right)$. Two parameters are of importance for the transport and decay of particulate organic matter to/in the deep ocean, namely the ratio of oxygen consumption to phosphate release during aer-

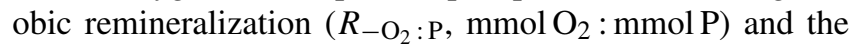
parameter for vertical increase in sinking speed of organic matter, $a\left(\mathrm{~d}^{-1}\right)$. Note that, as stated above, in the following, and during optimization, we express this last parameter through $b=r / a$, with $r$ held constant at $r=0.05 \mathrm{~d}^{-1}$.

For each parameter we initially chose a rather wide range of possible parameter values (Table 2). The lower value of $R_{-\mathrm{O}_{2}}:$ P was set to $150 \mathrm{mmol} \mathrm{O}_{2}: \mathrm{mmol} \mathrm{P}$ (Anderson, 1995), while its upper value is at the upper end of observed values (Boulahdid and Minster, 1989), and closer to values used in previous model studies (Paulmier et al., 2009). $b$ is allowed to vary between low values observed mainly in oxygen minimum zones (Van Mooy et al., 2002), and twice the global open ocean composite derived by Martin et al. (1987); its range is slightly larger than the range applied in previous modeling studies (Kwon and Primeau, 2006; Kriest and Oschlies, 2008; Kriest et al., 2012), or the range of $b$ determined from in situ observations (e.g., Martin et al., 1987; Buesseler et al., 2007). It agrees with the range of $b$ derived from indirect estimates of $b$ (Henson et al., 2012; Marsay et al., 2015).

Ranges of parameters related to surface processes were more difficult to assign. Due to the highly aggregated form of the organic biological components in the model, these parameters are supposed to reflect a variety of processes such as species shift and adaptation (e.g., half-saturation constants for nitrate uptake may vary over several orders of magnitude; see Collos et al., 2005). We therefore initially assigned very wide boundaries for $I_{\mathrm{c}}, K_{\mathrm{PHY}}, \mu_{\mathrm{ZOO}}$, and $\kappa_{\mathrm{ZOO}}$, which allow the optimization to pick parameters that virtually may shut down certain biological fluxes and processes. The choice of these wide boundaries, its consequences for optimization and model performance, and the effects of narrower boundaries will be examined and discussed below.

\subsection{Setup and performance of optimization}

Using the combined framework described above, i.e., TMM + MOPS + CMA-ES, we carried out five different full optimizations, with the aim of determining the four parameters related to surface biology and two parameters more closely tied to the deep biogeochemistry mentioned above. The experiments differ with respect to the observations used for the misfit function (model output, climatologies of observations), population size $\lambda$ of CMA-ES (10 or 20 individuals per generation), parameter boundaries, and the sampling strategy of CMA-ES. They are explained in detail below.

\subsubsection{TWIN experiment}

First we tested the ability of CMA-ES to recover known parameters of a model simulation that applied the same biogeochemical parameters as MOPS-RemHigh of Kriest and Oschlies (2015, setup "base", i.e., with a particle flux described by $b=0.858$, or $a=0.058275$, and a high affinity of oxic and suboxic remineralization to oxidants). This is done by optimization against its simulated annual average phosphate, nitrate, and oxygen of year 3000 . We refer to this experiment as "TWIN". TWIN applies rather wide boundaries for all parameters (see Table 2), and a population size for CMA-ES of $\lambda=10$, which was deemed sufficient for six parameters, given the default configuration of the CMA-ES (see above).

\subsubsection{Optimizations against observed tracers}

Four further optimizations were carried out against observations of annual mean phosphate, nitrate, and oxygen (Garcia et al., 2006a, b), gridded onto the model geometry. These are referred to as OBS-WIDE, OBS-WIDE-20, OBSNARR, and OBS-NARR-R. To investigate the robustness of CMA-ES with respect to different setups of the algorithm it- 
Table 3. Optimization results (evaluations, i.e., number of individuals, $\lambda$, times number of generations, $N$ ), best model misfit $M_{\text {opt }}$, optimum parameters, and their uncertainties. For each model and parameter, the first line gives the optimum parameter, followed by $p_{\text {min }}$ and maximum $p_{\max }$ of all individuals, for which the misfit $M_{i}$ is $\left(M_{i}-M_{\mathrm{opt}}\right) / M_{\mathrm{opt}} \leq 0.001$. The third line additionally presents in parentheses the percent of individuals for which this criterion holds, as well as the range of optimum parameters as a percent of the average parameter of the last generation. We also give the misfit and parameters of the reference run, against which the twin experiment was optimized.

\begin{tabular}{lcrrrrrrr}
\hline Experiment & $\lambda \times N$ & $M_{\mathrm{Opt}}$ & $R_{-\mathrm{O}_{2}: \mathrm{P}}$ & $I_{\mathrm{C}}$ & $K_{\mathrm{PHY}}$ & $\mu_{\mathrm{ZOO}}$ & $\kappa_{\mathrm{ZOO}}$ & $b$ \\
\hline Reference & 1 & 0.529 & 170.0 & 24.0 & 0.03125 & 2.0 & 3.2 & 0.858 \\
\hline TWIN & 2000 & 0.0003 & 170.0 & 24.0 & 0.034 & 2.0 & 3.20 & 0.858 \\
& & & 170 & 24 & $0.033-0.035$ & 2.0 & $3.19-3.20$ & 0.858 \\
& $(<1)$ & & $(<1)$ & $(<1)$ & $(5)$ & $(<1)$ & $(<1)$ & $(<1)$ \\
\hline OBS-WIDE & 950 & 0.477 & 179.5 & 48.0 & 0.12 & 0.28 & 6.15 & 1.10 \\
& & & $176-182$ & $46-49$ & $0.09-0.13$ & $0.24-0.32$ & $4.79-3.37$ & $1.08-1.12$ \\
& $(31)$ & & $(3)$ & $(6)$ & $(32)$ & $(28)$ & $(26)$ & $(4)$ \\
\hline OBS-WIDE-20 & 3460 & 0.450 & 167.7 & 9.9 & 0.5 & 2.05 & 5.83 & 1.34 \\
& & & $165-171$ & $9.6-10.8$ & $0.39-0.57$ & $2.00-2.52$ & $5.37-10.0$ & $1.31-1.37$ \\
& $(64)$ & & $(3)$ & $(12)$ & $(34)$ & $(25)$ & $(79)$ & $(5)$ \\
\hline OBS-NARR & 1820 & 0.450 & 167.0 & 9.7 & 0.5 & 1.89 & 4.57 & 1.34 \\
& & & $165-170$ & $9.0-10.3$ & $0.39-0.53$ & $1.57-2.02$ & $2.95-4.66$ & $1.30-1.36$ \\
& $(39)$ & & $(3)$ & $(14)$ & $(28)$ & $(23)$ & $(37)$ & $(4)$ \\
\hline OBS-NARR-R & 1400 & 0.450 & 166.7 & 9.6 & 0.5 & 1.76 & 3.82 & 1.34 \\
& & & $165-169$ & $8.7-10.1$ & $0.44-0.54$ & $1.57-1.79$ & $2.77-3.90$ & $1.31-1.36$ \\
& $(50)$ & & $(2)$ & $(14)$ & $(19)$ & $(13)$ & $(30)$ & $(3)$ \\
\hline
\end{tabular}

self, the experiments differ in the upper and lower boundaries of the search space for zooplankton parameters, the population size $\lambda$ of CMA-ES, and its sampling strategy. This is done in a stepwise fashion.

Experiment OBS-WIDE differs from TWIN only with respect to the observations that enter the misfit function. In OBS-WIDE we encountered an unlikely (with respect to biological tracer concentrations) solution, pointing towards a potential local minimum in the misfit function. We therefore set up two experiments to investigate strategies to improve the performance of CMA-ES with respect to more plausible solutions. The experiments both increase the search density in the parameter space with respect to OBS-WIDE. In experiment OBS-WIDE-20 search density is increased by doubling the population size of CMA-ES to $\lambda=20$. Otherwise, its setup is the same as OBS-WIDE. In experiment OBS-NARR we keep $\lambda=10$ of OBS-WIDE, but restrict the boundaries for zooplankton parameters to $\pm 50 \%$ of the value of the reference run of MOPS.

Because optimization OBS-NARR showed the best results with respect to misfit function, biogeochemical fluxes, and optimization performance (see below; Tables 3 and 4), in experiment "OBS-NARR-R" we finally evaluate the robustness of optimization OBS-NARR by repeating this optimization with a different random selection of the parameter values from the distribution calculated by CMA-ES.

\subsubsection{Performance}

The internal termination criterion of CMA-ES was reached after 95, 173, 182, and 140 generations for OBS-WIDE, OBS-WIDE-20, OBS-NARR, and OBS-NARR-R, respectively. For the twin experiment, we restricted the maximum number of generations to 200 , at which TWIN had approached the target parameters, the misfit declined to $<0.0004$ (i.e., on average less that $0.2 \%$ of global mean tracer concentrations; see Eq. 4) and fitness variance declined to $<10^{-9}$. As presented above, in each "generation" we computed 10 (20) different "individuals" (model simulations over 3000 years) in parallel. One simulation of each generation on average took $\approx 1.25 \mathrm{~h}$, on 40 (80) nodes of Intel Xeon IvyBridge or Intel Xeon Haswell at the North-German Supercomputing Alliance (HLRN). We note that tests on either hardware (two iterations of the coupled code, started from generations 80 and 160 of experiment TWIN) did not reveal any differences in the estimated fitness. The CMA-ES - which, due to its very short runtime, is not parallelized was always computed on one core of Intel Xeon IvyBridge.

\section{Results}

\subsection{Twin experiment (TWIN)}

The optimization starts with a wide range of potential parameters (see Fig. 3), with individual parameters sometimes even 
Table 4. Global annual fluxes of primary production (PP), grazing (GRAZ), aerobic and anaerobic remineralization of detritus and DOM to nutrients (REM), excretion by zooplankton (EXCR) export production $\left(F_{120}\right.$, flux through $\left.120 \mathrm{~m}\right)$, flux through $2030 \mathrm{~m}\left(F_{2030}\right)$, and benthic burial (BUR), in $\mathrm{Pg} \mathrm{N}_{\text {year }}{ }^{-1}$, for the reference experiment, OBS-WIDE, OBS-WIDE-20, and OBS-NARR (two repeated experiments with different configurations of CMA-ES). We also show some globally derived, observed estimates. Conversion between different elements was carried out via $\mathrm{N}: \mathrm{P}=16$ and $\mathrm{C}: \mathrm{P}=122$.

\begin{tabular}{lrrrrrrr}
\hline Experiment & PP & GRAZ & REM & EXCR & $F_{120}$ & $F_{2030}$ & BUR \\
\hline Reference & 5.44 & 3.52 & 4.72 & 0.80 & 0.92 & 0.11 & 0.05 \\
OBS-WIDE & 6.20 & 1.24 & 5.94 & 0.25 & 0.81 & 0.06 & 0.02 \\
OBS-WIDE-20 & 7.45 & 4.68 & 6.66 & 1.00 & 1.10 & 0.06 & 0.02 \\
OBS-NARR & 7.52 & 4.74 & 6.65 & 1.10 & 1.10 & 0.06 & 0.02 \\
OBS-NARR-R & 7.58 & 4.77 & 6.65 & 1.19 & 1.10 & 0.06 & 0.02 \\
Observed* $^{*}$ & $7.68-8.09$ & $4.79,5.71$ & - & - & $0.29-1.53$ & $0.03-0.07$ & 0.02 \\
\hline
\end{tabular}

* Observed fluxes are from Carr et al. (2006, primary production), Honjo et al. (2008, particle flux), Lutz et al. (2007, particle flux), Dunne et al. (2007, particle flux), Schmoker et al. (2013, primary production, zooplankton grazing excluding/including mesozooplankton grazing) and Wallmann (2010, burial; without shelf and slope region).
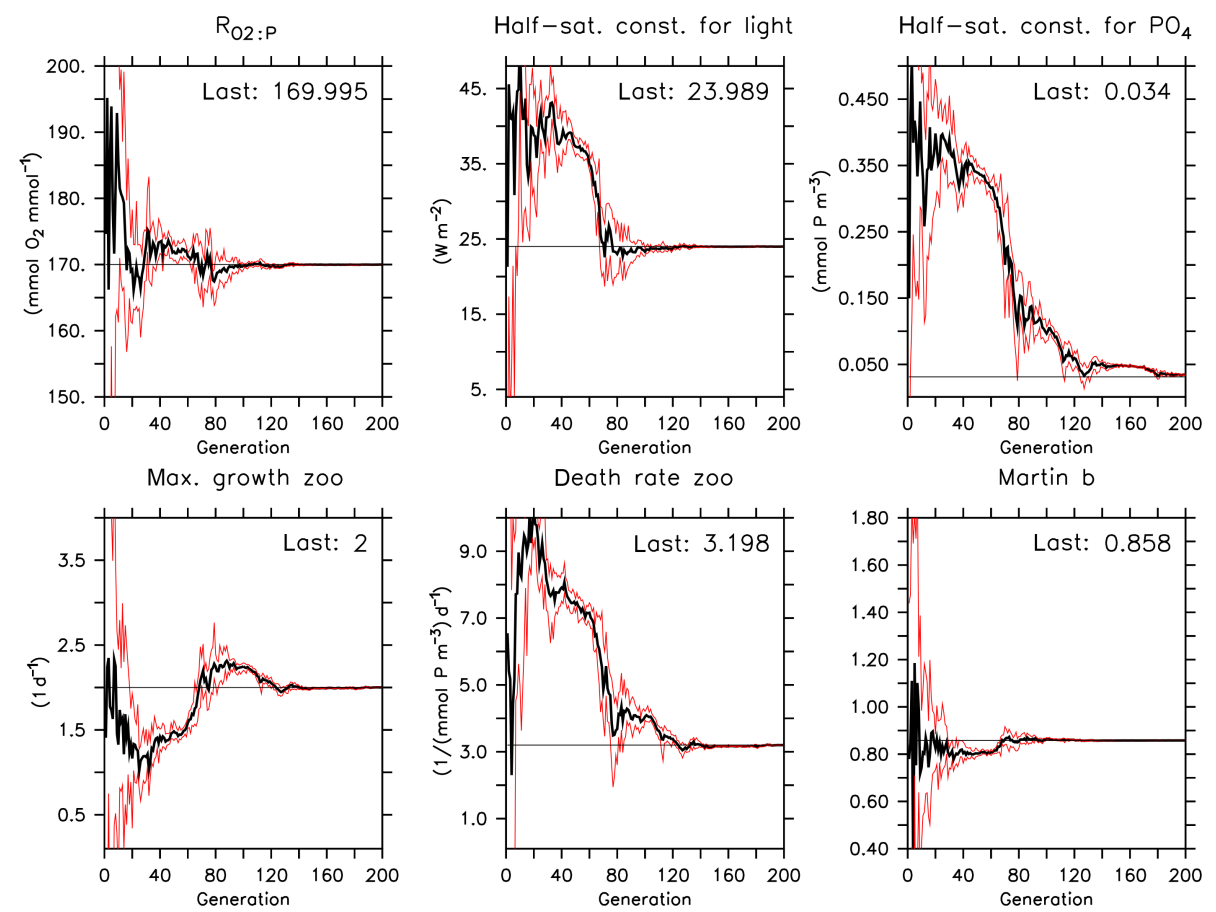

Figure 3. Optimization trajectory for six parameters of the twin experiment. The thick black line shows the average parameter of all 10 individuals of a generation. Red lines indicate their maximum and minimum parameter values. Horizontal black lines indicate the target parameter. Note that we restrict the $y$-axis to the maximum and minimum boundaries.

exceeding the prescribed boundaries. This results in high maximum and minimum misfits (Fig. 4), and this high variability is maintained over about $10-20$ generations. The trajectory of transient average parameter values and their variance depend strongly on the parameter itself: while the two parameters associated with rather long timescales, namely the stoichiometric ratio $R_{-\mathrm{O}_{2}: \mathrm{P}}$ and exponent $b$ describing particle sinking, approach their target values quite early (about generation 20-40), parameters associated with surface biogeochemistry stay far away from their target value for $\approx 80$ generations $\left(I_{\mathrm{c}}, K_{\mathrm{PHY}}, \kappa_{\mathrm{ZOO}}\right)$ or oscillate around it $\left(\mu_{\mathrm{ZOO}}\right)$. After $\approx 160$ generations, most of the parameters reached their target value, the exception being the halfsaturation constant of phytoplankton for phosphate uptake, $K_{\text {PHY }}$ (Table 3). This parameter still shows considerable variability at the end of the optimization (generation 200), although by that time is it quite close to the - rather low target value.

The misfit function, its variance, and the parameter variance do not decrease monotonically throughout the optimization trajectory. In particular, after an initial decline over ca. 60 generations, parameter and misfit variance increase again. 

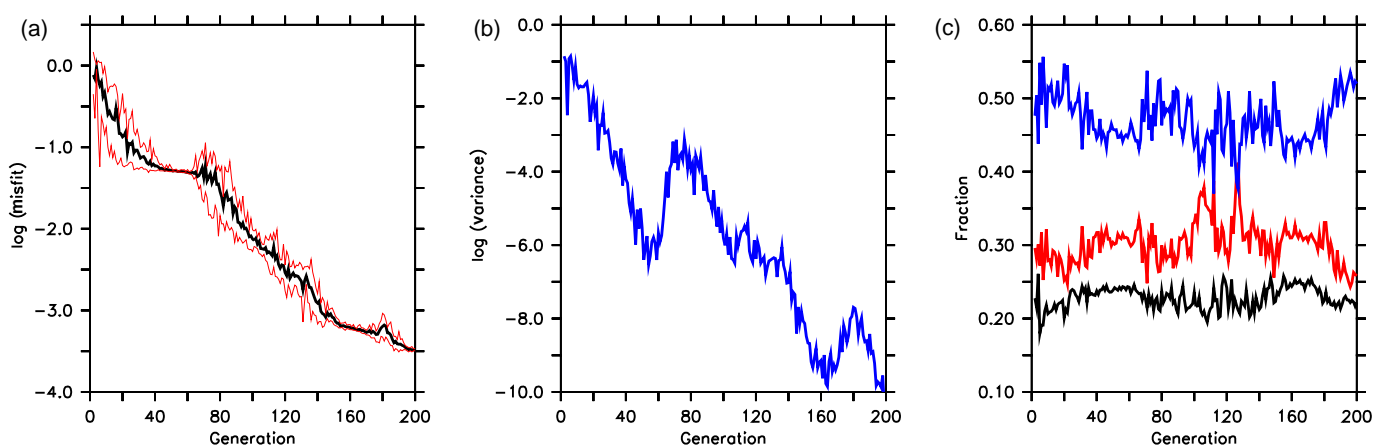

Figure 4. Model misfit, its variance, calculated from individuals of each population (both transformed logarithmically by log 10 ) and components of the twin experiment. Left panel: the thick black line shows the average misfit of all 10 individuals of a generation. Red lines indicate the maximum and minimum misfit. Mid panel: variance of misfit. Right panel: contribution of each component of the misfit function. Blue: oxygen. Red: nitrate. Black: phosphate.
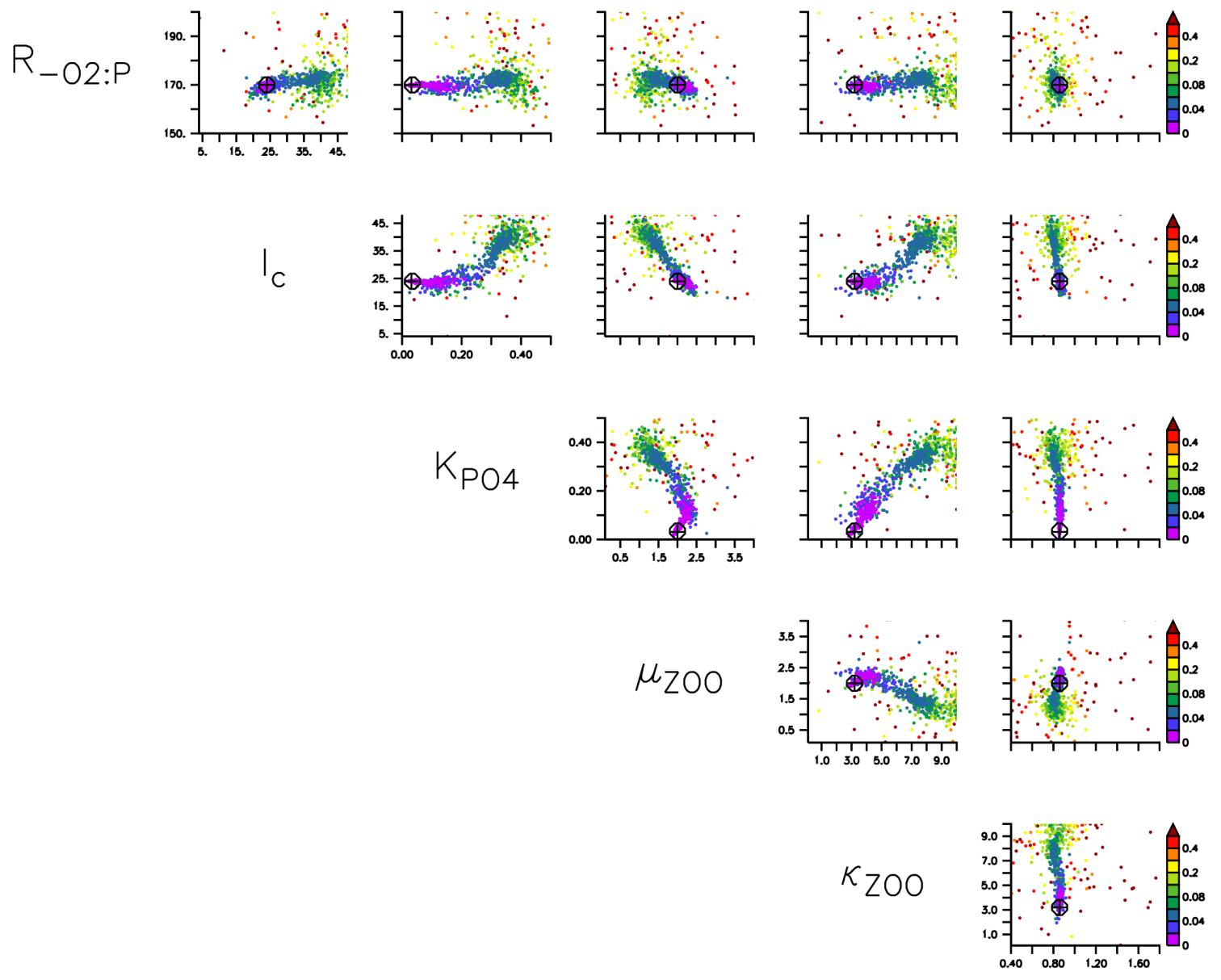

Figure 5. Model misfit, plotted for each pair of parameter combinations of the twin experiment. Color indicates misfit (see the color bars on the right). A cross indicates the target value, i.e., the value of the reference experiment. A circle indicates the parameter of one individual of the last generation. Note that for better visibility we restrict the parameter range to its boundaries (see Table 2). 

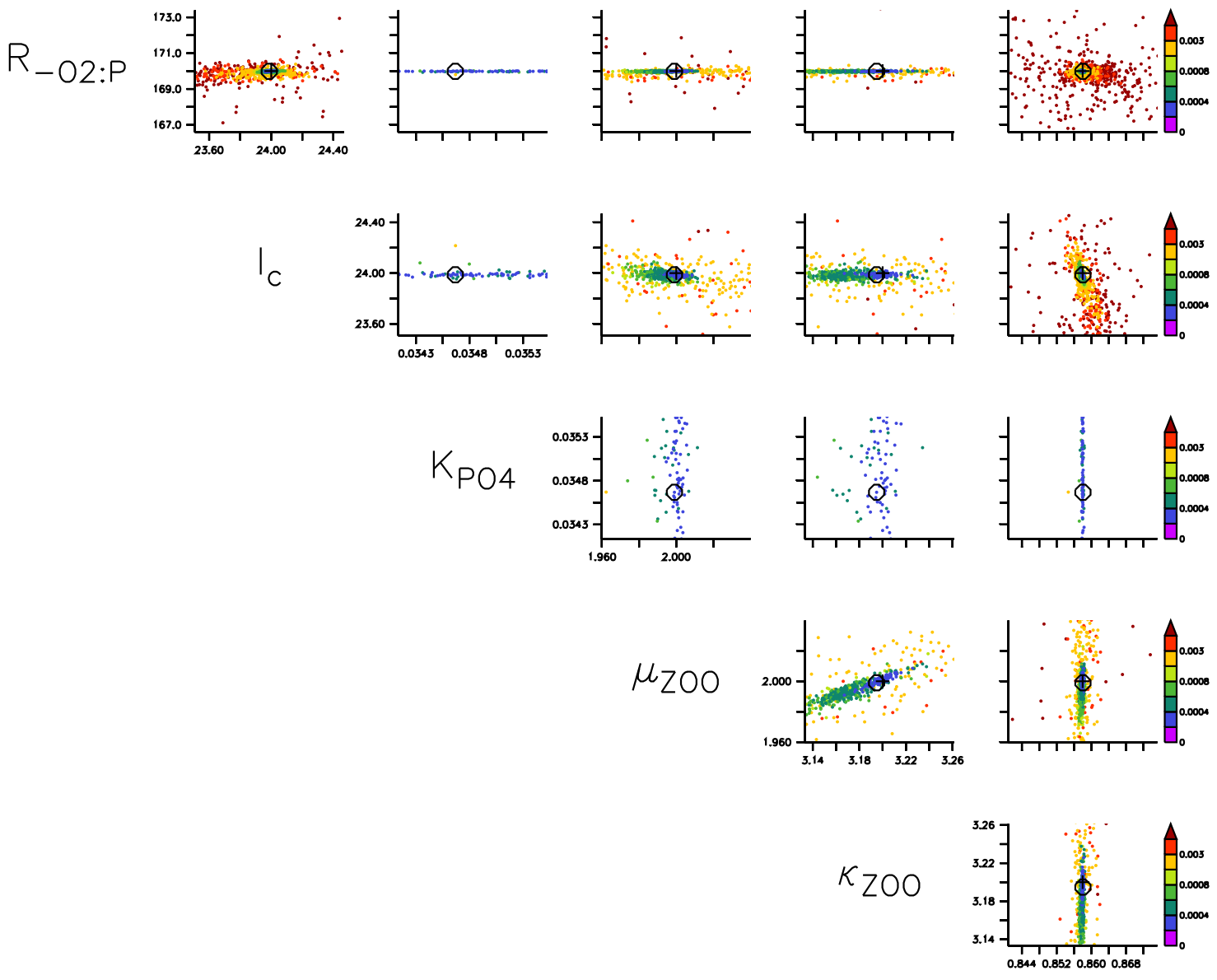

$b$

Figure 6. As Fig. 5, but only plotted for a region $\pm 2 \%$ around the average parameter value of the last generation, regardless of generation and associated misfit. Note that these parameters can have occurred early in the optimization and even be associated with a large misfit (that would arise from at least one of the other parameters, causing a large misfit). Note that the color scale is different than in Fig. 5 .

Further increases in variance can be seen around generation 100 , and, at the end, when the algorithm widens its search area again, probably in search of an optimal $K_{\mathrm{PHY}}$. It seems encouraging that the algorithm does not get stuck in a local minimum, but, at the expense of deterioration of the misfit, continues to search for an even better parameter set.

The largest fraction of the misfit function is related to oxygen, followed by the misfit to nitrate, and then phosphate. The dominance of oxygen and nitrate is not surprising, as these tracers are not conservative; i.e., their global inventory might change due to air-sea gas exchange, denitrification, and nitrogen fixation (see also Kriest and Oschlies, 2015), so that the model may not only err with respect to the spatial distribution of these tracers, but also with respect to their global mean concentration.

In Fig. 5 we finally exploit the shape of the misfit function, shown on a color scale for every two pairs of parameters. As can be seen from the misfit plotted against $R_{-\mathrm{O}_{2}: \mathrm{P}}$ and $b$ (upper right corner), these two parameters are quite well constrained, with a very well-defined minimum around the target value. All other parameters show more or less elongated search "canyons". Much of the algorithm search starts away from the target value; however, the algorithm finally manages to approach the target value even when the search path is not straight, but curved in the two-dimensional projections of the parameter space. Further, even when the algorithm exceeds the target value (e.g., for the maximum growth rate of zooplankton, $\mu_{\mathrm{ZOO}}$; lower right corner), despite the already low misfit function, the algorithm finally returns to the somewhat lower value (compare also to Fig. 3, lower left panel).

Summarizing, CMA-ES seems capable of dealing even with our irregular search landscape, when iterated for a long enough time and with a sufficiently large population size. A problem remains with regards to the half-saturation constant of phytoplankton for phosphate uptake: zooming into the scatter plot presented in Fig. 5 reveals that for this pa- 

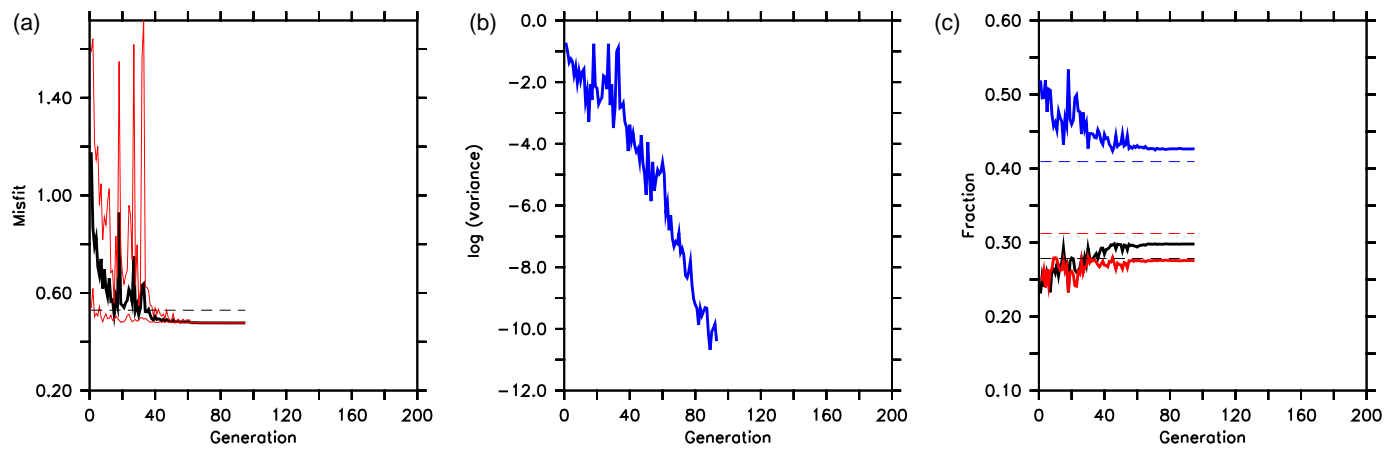

Figure 7. As Fig. 4, but for optimization OBS-WIDE. Note that in the left plot, we now show the raw value of the misfit function (not log transformed). The optimization finished at generation 95 .

rameter the search landscape becomes quite uninformative (Fig. 6), with similar misfits around $\pm 2 \%$ of its last value. Thus, a low misfit can be achieved within a wide range of this parameter.

One reason for this low sensitivity of the misfit function to $K_{\mathrm{PHY}}$ may be found in the fact that, in the twin, against which the model is optimized, only very few $(1 \%)$ phosphate values are at or below the target value of $K_{\mathrm{PHY}}=$ $0.03125 \mathrm{mmol} \mathrm{P} \mathrm{m}^{-3}$. Therefore, besides the dominance of oxygen in the misfit function (Fig. 4), the misfit function is further dominated by phosphate concentrations outside the oligotrophic surface regions, rendering it quite insensitive to changes in the half-saturation constant at low values. In addition, a closer look at the misfit topography (Fig. 5) points towards a potential correlation of $\mu_{\mathrm{ZOO}}$ and $\kappa_{\mathrm{ZOOO}}$, which may complicate the algorithm's search for an optimum set of parameters, thereby slowing down its convergence.

\subsection{Optimization against observed nutrients and oxygen distributions}

\subsubsection{Wide boundary constraints for zooplankton (OBS-WIDE, OBS-WIDE-20)}

When optimizing the model against observed concentrations with exactly the same setup as for experiment TWIN, optimization OBS-WIDE reaches the internal termination criterion of the CMA-ES at generation 95. Instead of declining exponentially towards zero, the misfit only declines from an average initial value of $\approx 0.8$ to 0.477 (Fig. 7 , Table 3), i.e., only slightly less than the misfit of the reference run (0.529). Also, the variance of misfit as well as that of the parameters show a more or less gradual decline, without any intermittent increase (see the Supplement). Another notable difference to TWIN is the higher contribution of phosphate to the misfit function (Fig. 7).

Some parameters diverge strongly from those of the reference run. In particular, the phytoplankton's half-saturation constant for light, $I_{\mathrm{c}}$, increases strongly up to its upper boundary (Fig. 8; Table 3; see also the Supplement for a plot of the topography of the misfit function). However, the stronger light limitation of phytoplankton growth is counteracted by a strong decrease in zooplankton growth rate, $\mu_{\mathrm{ZOO}}$, and a strong increase in its quadratic mortality rate, $\kappa$ ZOO. As a consequence, average and maximum zooplankton concentrations are $<25$ and $<50 \%$ of that of the reference run in the surface layer (Fig. 9), while phytoplankton is strongly increased, when compared to the reference run. Most likely because the zooplankton-detritus pathway is nearly shut off, DOM concentrations are strongly increased. The reorganization of the pelagic food web in this optimized model scenario is reflected in the global annual biogeochemical fluxes: primary production is enhanced by almost $14 \%$, but loss through grazing is reduced to about $1 / 3$ of that of the reference run (Table 4). As a consequence, the largest fraction of recycling is through remineralization of detritus and DOM ( $>95 \%$ of annual production), and only $4 \%$ through zooplankton excretion, while in the reference run zooplankton recycles almost $15 \%$ of annual production. Due to the reduced particle sinking speed, shallow $(130 \mathrm{~m})$ and deep $(2030 \mathrm{~m})$ particle fluxes are reduced, as is benthic burial. While some of the simulated fluxes are within the observed estimates, overly low zooplankton concentration, as well as the resulting low zooplankton grazing, are far outside observed estimates (see Table 4).

Therefore, although optimization OBS-WIDE against observations has decreased the misfit to observations to $\approx 90 \%$ of that of the (subjectively tuned) reference run, the outcome is not overly satisfying with respect to the optimized parameters and the resulting dynamical behavior of the model. Obviously, the very wide boundary constraints we chose for the zooplankton parameters led to a solution where zooplankton is almost dead - a statistically optimal but biologically meaningless solution.

To examine whether this optimization became trapped in a local minimum, in experiment OBS-WIDE-20 we increased the population size of CMA-ES from $\lambda=10$ to $\lambda=20$. Due to a larger population, in this optimization the variability of fitness (Fig. 10) and parameter values (Fig. 11) is maintained 

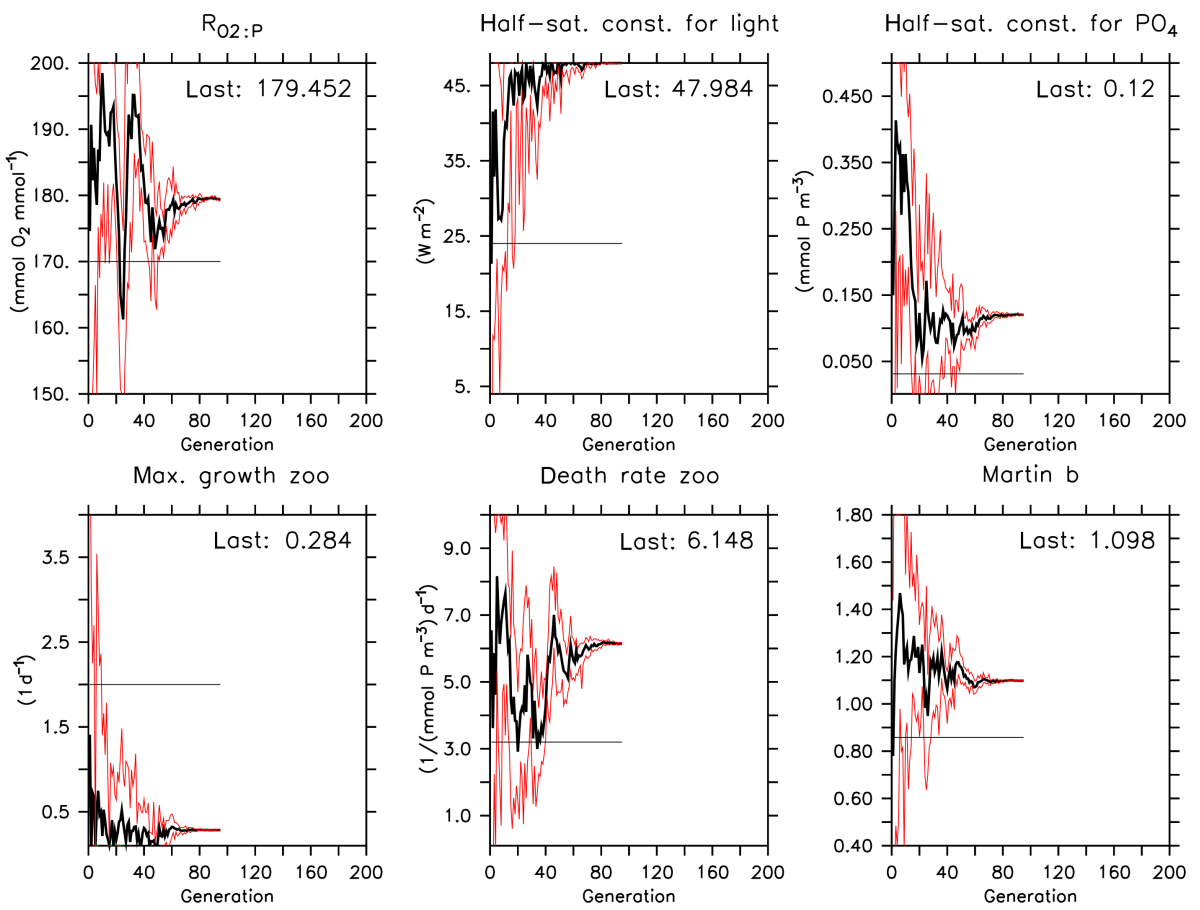

Figure 8. As Fig. 3, but for optimization OBS-WIDE. The optimization finished at generation 95.

over a longer period, again, as for optimization TWIN, with intermittent increases in variance during the course of the optimization. Most importantly, using the setup of OBS-WIDE20 , the optimization finds very different parameters for many of the biogeochemical components.

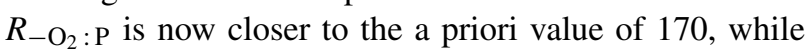
optimal $b$ has increased considerably to $b=1.34$ (Table 3 ). The largest difference to both the reference run as well as optimization OBS-WIDE occurs for the four biogeochemical parameters that are more closely tied to surface processes: $I_{\mathrm{C}}$ decreases to less than $50 \%$ of its a priori value, while $K_{\mathrm{PHY}}$ is at its upper boundary of $0.5 \mathrm{mmol} \mathrm{P} \mathrm{m}^{-3}$. Encouragingly, zooplankton parameters are now such that zooplankton is viable (Fig. 9). Its maximum growth rate is very close to the a priori value of $2 \mathrm{~d}^{-1}$. Its mortality rate is still quite high; however, because of its high growth rate, zooplankton plays a considerable role in the pelagic nitrogen budget, with global fluxes much closer to the observed ones than for optimization OBS-WIDE (Table 4). The topography of the - rather dense - scan of the parameter space of OBS-WIDE-20 (Fig. 12) points towards a potential correlation between $K_{\mathrm{PHY}}, \mu_{\mathrm{ZOO}}$, and $\kappa$ ZOO. In this projection, low misfit values occur along a concomitant increase in $K_{\mathrm{PHY}}$ with either $\mu_{\mathrm{ZOO}}$ or $\kappa_{\mathrm{ZOO}}$. This is also reflected in the high level of parametric uncertainty, as revealed by a large range of parameter values in the vicinity of the optimum (Table 3 ).

Summarizing, using a larger population size and thus a denser scan of the parameter space (see Fig. 12), CMA-ES has found a better solution with respect to the misfit function (see Table 3) as well as a closer fit to biogeochemical fluxes and more plausible biological patterns.

\subsubsection{Narrow boundary constraints for zooplankton (OBS-NARR and OBS-NARR-R)}

Optimizations with a population size of $\lambda=20$, as for OBSWIDE-20, are computationally quite expensive, especially when iterated over a large number of generations (Table 3). Via the quite wide boundary constraints for zooplankton parameters, we have assumed to have almost no knowledge about zooplankton. In the following two sensitivity experiments we examine the impact of this assumption on optimization performance, by restricting zooplankton parameters to a narrower range. These experiments are again carried out with a population size of $\lambda=10$.

To enforce live zooplankton, we restricted the range of zooplankton parameters to $\pm 50 \%$ of their reference value. This results indeed in a solution with organic tracer concentrations close to that of the reference run or OBS-WIDE-20 (Fig. 9). After 182 generations, the algorithm terminates with a misfit of 0.45 (Fig. 13), i.e., better than experiment OBSWIDE, but the same as for optimization OBS-WIDE-20 (Table 3). As in TWIN and OBS-WIDE-20, misfit variance shows intermittent increases, and the contribution of nitrate to the misfit function dominates over that of phosphate. Likewise, resulting optimal parameter values are quite close to those of OBS-WIDE-20 (Table 3). Thus, OBS-NARROW is more similar to OBS-WIDE-20 than to OBS-WIDE, demon- 

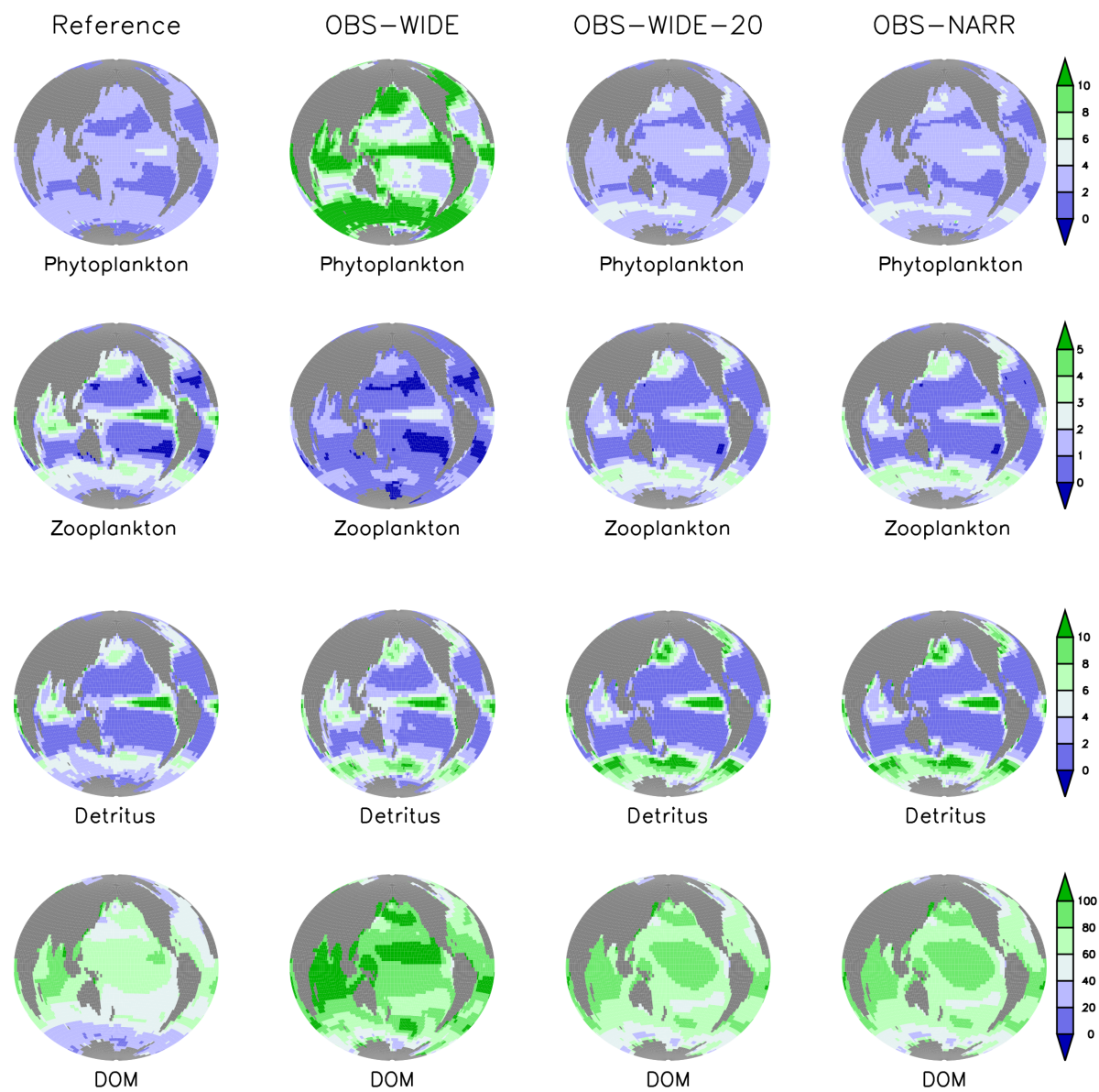

Figure 9. Surface (first) layer concentrations (in $\mathrm{mmol} \mathrm{C}^{-3}$, converted via a $\mathrm{C}: \mathrm{P}$ ratio of 122) for phytoplankton, zooplankton, detritus, and DOM for the reference run and optimizations OBS-WIDE, OBS-WIDE-20, and OBS-NARR.
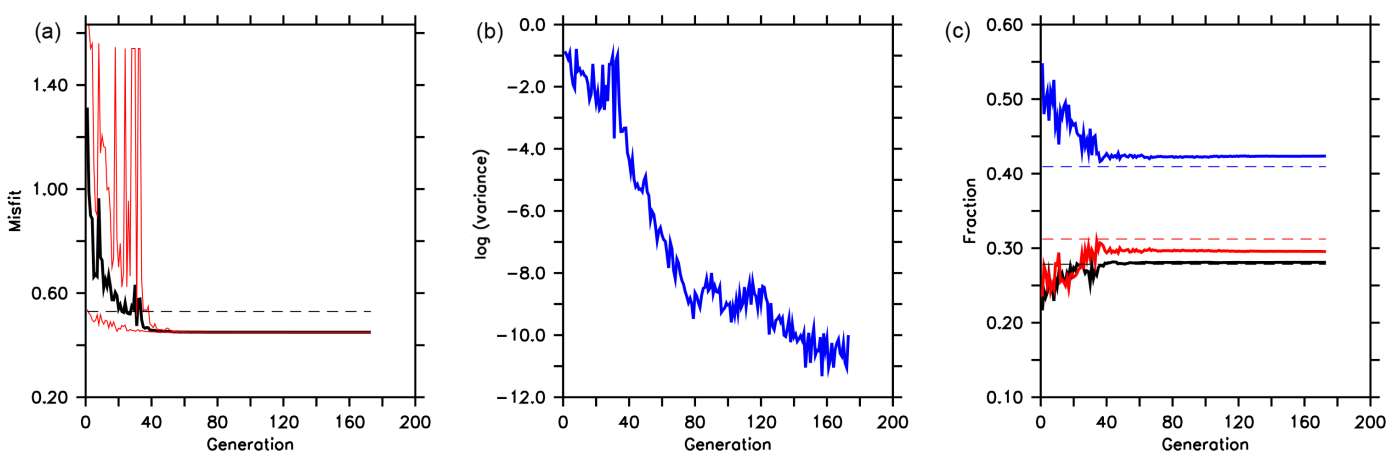

Figure 10. As Fig. 7, but for optimization OBS-WIDE-20. The optimization finished at generation 173.

strating the importance of good a priori knowledge about parameter values.

As for OBS-WIDE-20, the quadratic mortality of zooplankton, $\kappa_{\mathrm{ZOO}}$, and the half-saturation constant of phosphate uptake for phytoplankton, $K_{\mathrm{PHY}}$, show a strong increase, the latter up to its upper prescribed boundary, which may be interpreted as an attempt of the algorithm to force the model towards higher surface nutrient concentrations in the subtrop- ical gyres. A reduced half-saturation constant for light, on the other hand, counteracts the grazing pressure exerted by zooplankton, particularly in the high latitudes. Most likely because of increased detritus production by zooplankton and thus increased export from the surface layer (Table 4) particle flux to the deep ocean is reduced by an increase in $b$, i.e., a relatively slow particle sinking speed. 

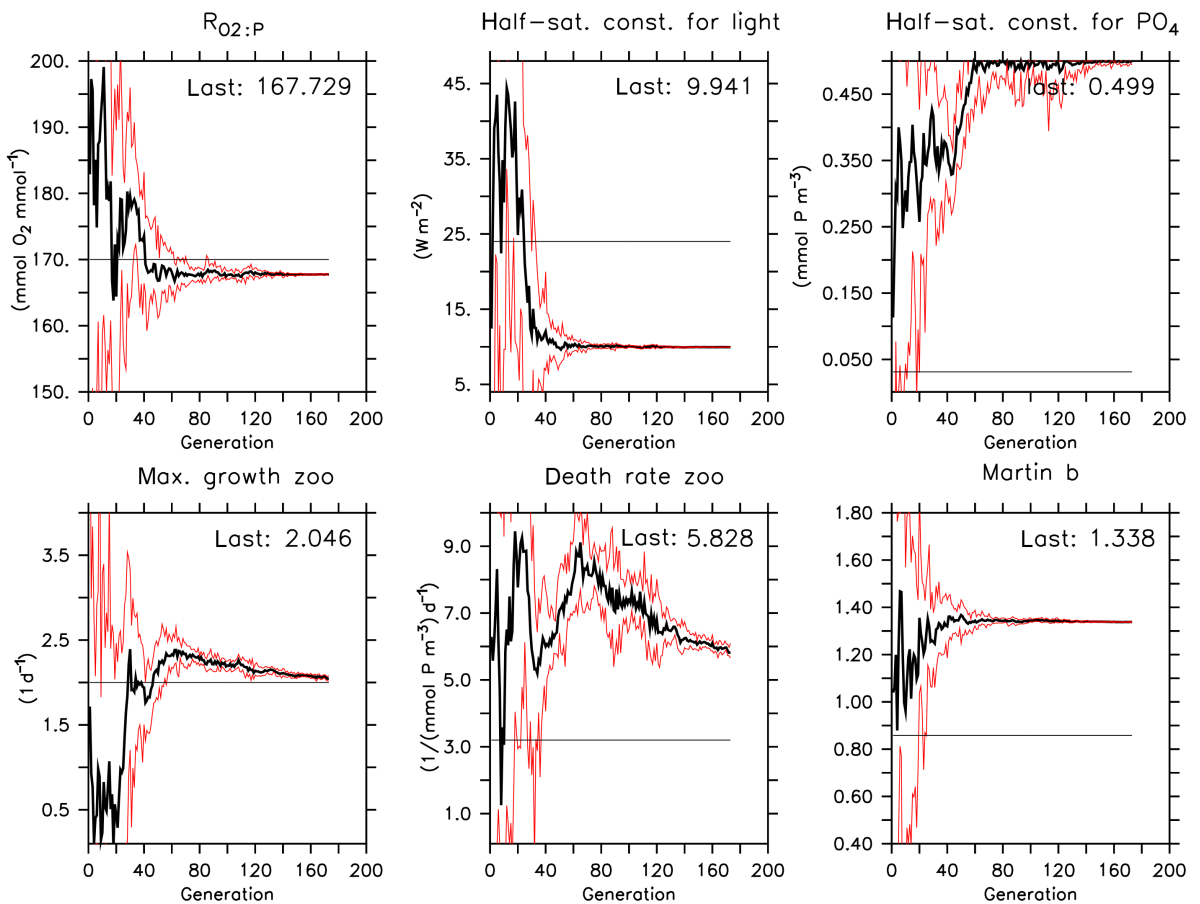

Figure 11. As Fig. 8, but for optimization OBS-WIDE-20. The optimization finished at generation 173.

A closer look at the topography of the misfit function shows that the misfit is quite insensitive to changes in some parameters (Fig. 15; see the Supplement for a detailed plot of misfit topography around $\pm 2 \%$ of the optimal parameters). While the parameters $R_{-\mathrm{O}_{2}: \mathrm{P}}$ and $b$, which tend to exert an influence on large temporal and spatial scales, are again quite well constrained, many of the surface-related parameters that act on smaller timescales, such as $K_{\mathrm{PHY}}$, show a wide scatter across the parameter space (see also Table 3 ), with very little differences in the misfit function.

However, variations in parameters after $\approx 40$ generations do not strongly improve the model fit to observations (Figs. 13 and 14). The rather constant misfit after generation 40 is quite surprising, given that some parameters still show some significant excursions after that time, indicating that - as already shown before - the misfit function is quite uninformative about these parameters. This insensitivity of inorganic tracers is also illustrated in Fig. 16, which shows the deviation of vertically integrated tracers from observations, plotted for individuals of three different generations of OBS-NARR (see also the blue vertical lines in Fig. 14). The parameters of these individuals differ mainly with respect to their combination of $K_{\mathrm{PHY}}$ and $\kappa_{\mathrm{ZOO}}$. While the reference run applies very low $K_{\mathrm{PHY}}=0.03125 \mathrm{mmol} \mathrm{P} \mathrm{m}^{-3}$ and moderate $\kappa_{\mathrm{ZOO}}=3.2\left(\mathrm{mmol} \mathrm{P} \mathrm{m}^{-3}\right)^{-1} \mathrm{~d}^{-1}$, individuals of the optimization are characterized by medium (generation 61) to high (generations 110 and 182) $K_{\mathrm{PHY}}$, and moderate (generations 61 and 110) and high (generation 182) $\kappa$ ZOO (see also the blue vertical lines in Fig. 14). All individuals differ from the reference run, yet the difference between them is almost not visible in the simulated tracer distributions. Thus, annual mean tracer concentrations on a global scale do not seem to suffice in constraining some of the parameters related to the very dynamic biological turnover at the sea surface, leading to a large parametric uncertainty (Table 3), possibly amplified by correlation between these three parameters.

Except for deep particle fluxes, all biogeochemical fluxes are increased compared to the reference run or experiment OBS-WIDE, but similar to that of OBS-WIDE-20 (Table 4). Therefore, although the misfit function so far only optimized towards inorganic constituents, the optimized model with narrow zooplankton parameter boundaries shows a much better fit to observed global fluxes to primary production, zooplankton grazing, shallow and deep particle flux, and benthic burial. The seemingly better dynamical biogeochemical behavior of this model setup gives some confidence that the model's fit to inorganic tracers is not improved at the cost of any other tracer.

Repeating optimization OBS-NARR with a different random selection of parameters from the parameter distribution in each generation (OBS-NARR-R) yields the same, or very similar, best values for most of the parameters (see Table 2), the exception being the two zooplankton parameters, $\mu_{\mathrm{ZOO}}$ and $\kappa_{\mathrm{ZOOO}}$. These two parameters of OBS-NARR-R are $7 \%$ $\left(\mu_{\mathrm{ZOO}}\right)$ and $16 \%(\kappa \mathrm{zOO})$ lower than in OBS-NARR; however, the misfit of both optimizations is the same (0.45). The low sensitivity of the misfit function to zooplankton parameters is mirrored in similar nutrient and oxygen distributions 

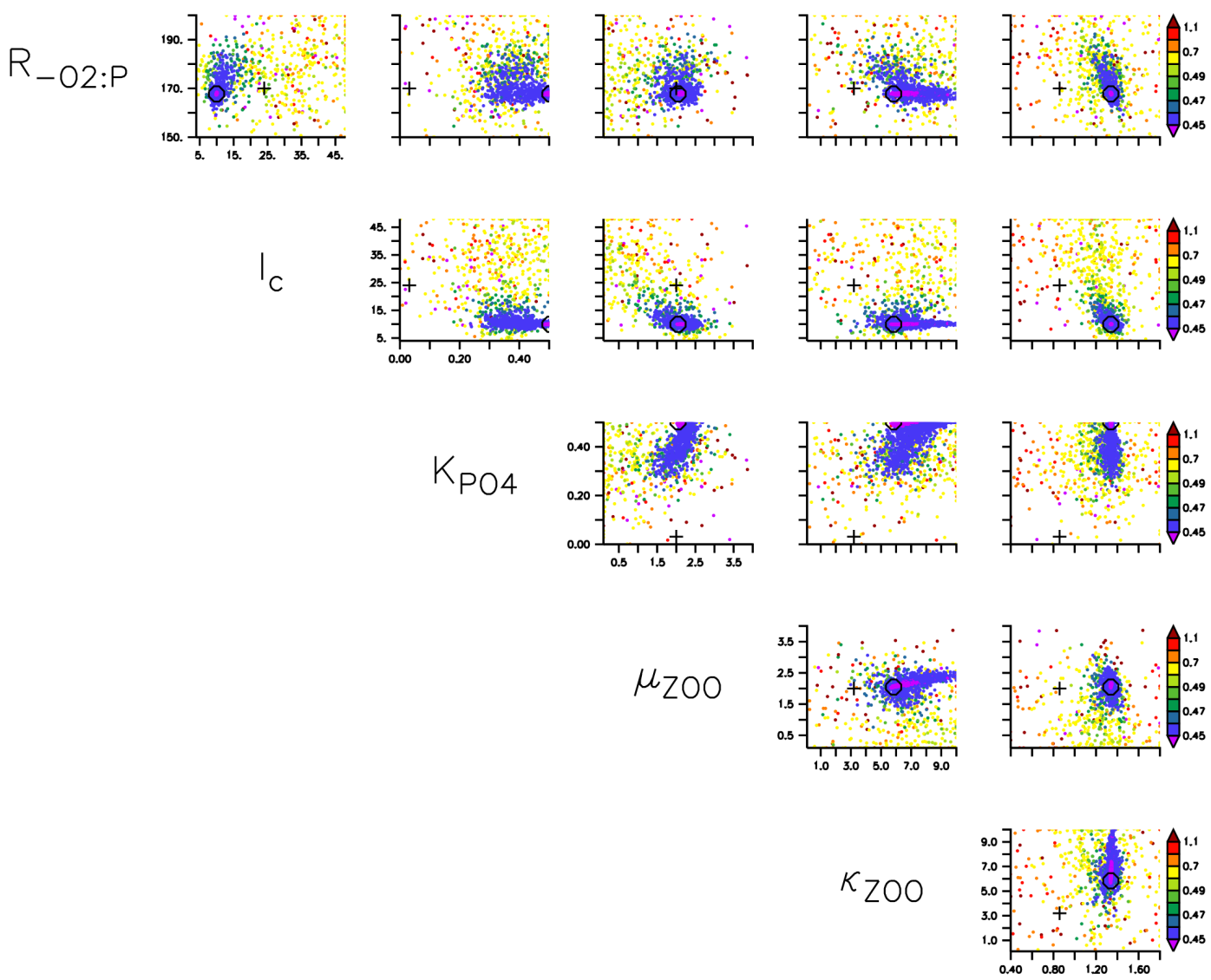

b

Figure 12. As Fig. 5, but for optimization OBS-WIDE-20. Note that the color scale differs from that of Fig. 5.
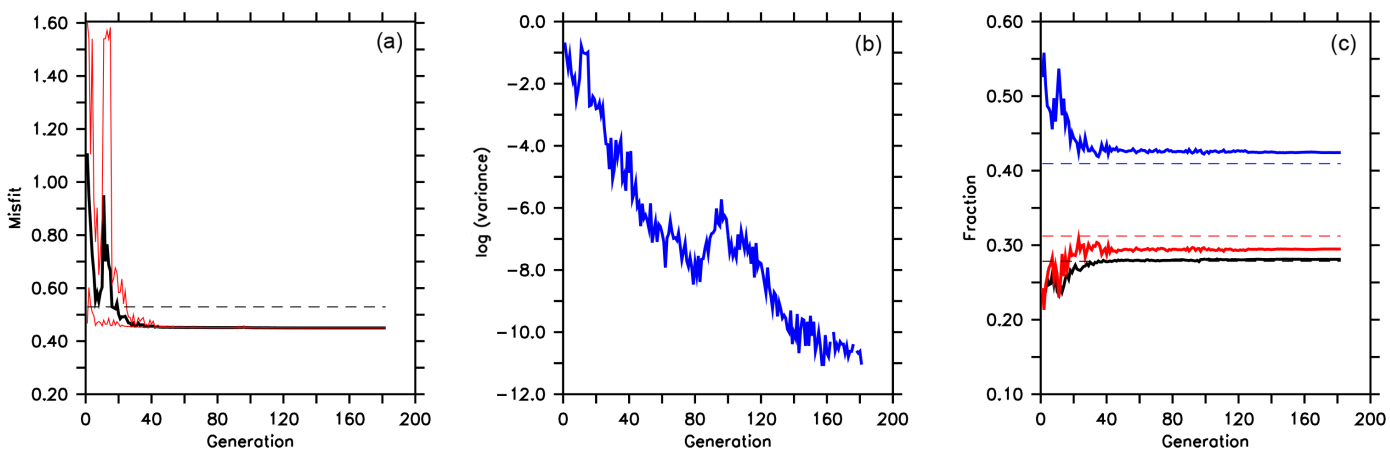

Figure 13. As Fig. 10, but for optimization OBS-NARR. The optimization finished at generation 182. 

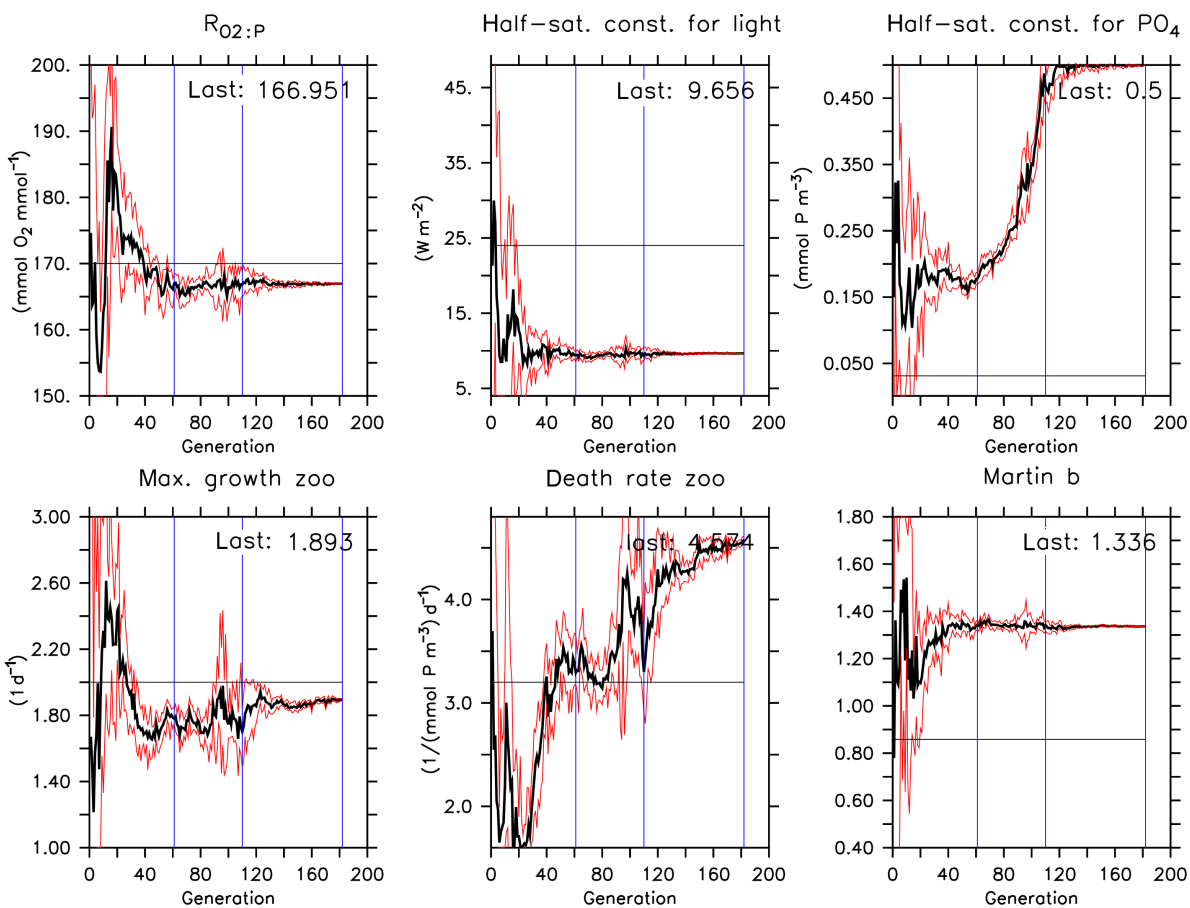

Figure 14. As Fig. 11, but for optimization OBS-NARR. The optimization finished at generation 182. Vertical blue lines indicate generation, for which we also present deviations from observation of vertically integrated nutrients and oxygen from Fig. 16.

(see the Supplement) and almost identical biogeochemical fluxes (see Table 4).

\section{Discussion}

\subsection{Computational performance}

Our results suggest that the CMA-ES optimization algorithm performs well, particularly for the twin experiment, even though the parameters to be estimated involve diverse temporal and spatial scales. CMA-ES manages to set up curved search paths in parameter space, and therefore is capable of approaching an optimum within a rather complex topography of the misfit function. Its sometimes elongated and/or curved shape resembles many of those resulting from earlier one-dimensional (Athias et al., 2000; Schartau et al., 2001; Schartau and Oschlies, 2003a; Ward, 2009) or threedimensional (Kwon and Primeau, 2006, 2008) optimizations of marine biogeochemical models. However, when imposing wide boundary constraints for zooplankton parameters, OBS-WIDE becomes trapped in a local minimum; only with a larger population size or narrower parameter boundaries do we find a solution that results in realistic concentrations and fluxes of all components. Clearly, the number of experiments conducted here is too small to make statistically significant statements about the optimizers' exploration capability with respect to the population size. But similar to other population based heuristics, examinations with multi- modal test functions have given evidence that larger populations increase CMA-ES' chances of finding good local optima (or even a global optimum; Hansen and Kern, 2004). It remains to be investigated whether different configurations of the CMA-ES, or a different optimization algorithm, e.g., gradient based methods or evolutionary algorithms, perform better or worse with respect to the number of model evaluations required or their ability to avoid local minima (see also Athias et al., 2000). However, there is some indication that genetic algorithms perform better with respect to a rough topography of the misfit function, when compared to a variational adjoint method, with an otherwise equally good fit to marine biogeochemical observations (Ward et al., 2010).

As the computational effort remains a challenge in parameter optimization of global ocean BGC models, further possibilities to accelerate model evaluations within the optimization process are desirable. Surrogate-assisted approaches use meta-models to approximate model evaluations within optimization (Priess et al., 2013). They are becoming practice within evolutionary frameworks coping with computationally expensive model functions (Jin, 2011). It should be worth considering surrogate approaches with CMA-ES as investigated in Kern et al. (2006), Auger et al. (2013), and Loshchilov et al. (2012). A general approach with EA and EDA frameworks is to prematurely abort the fitness calculation after detecting that the corresponding individual will not be better than the worst member of the current population. We can benefit from such short-cut fitness computation if the 

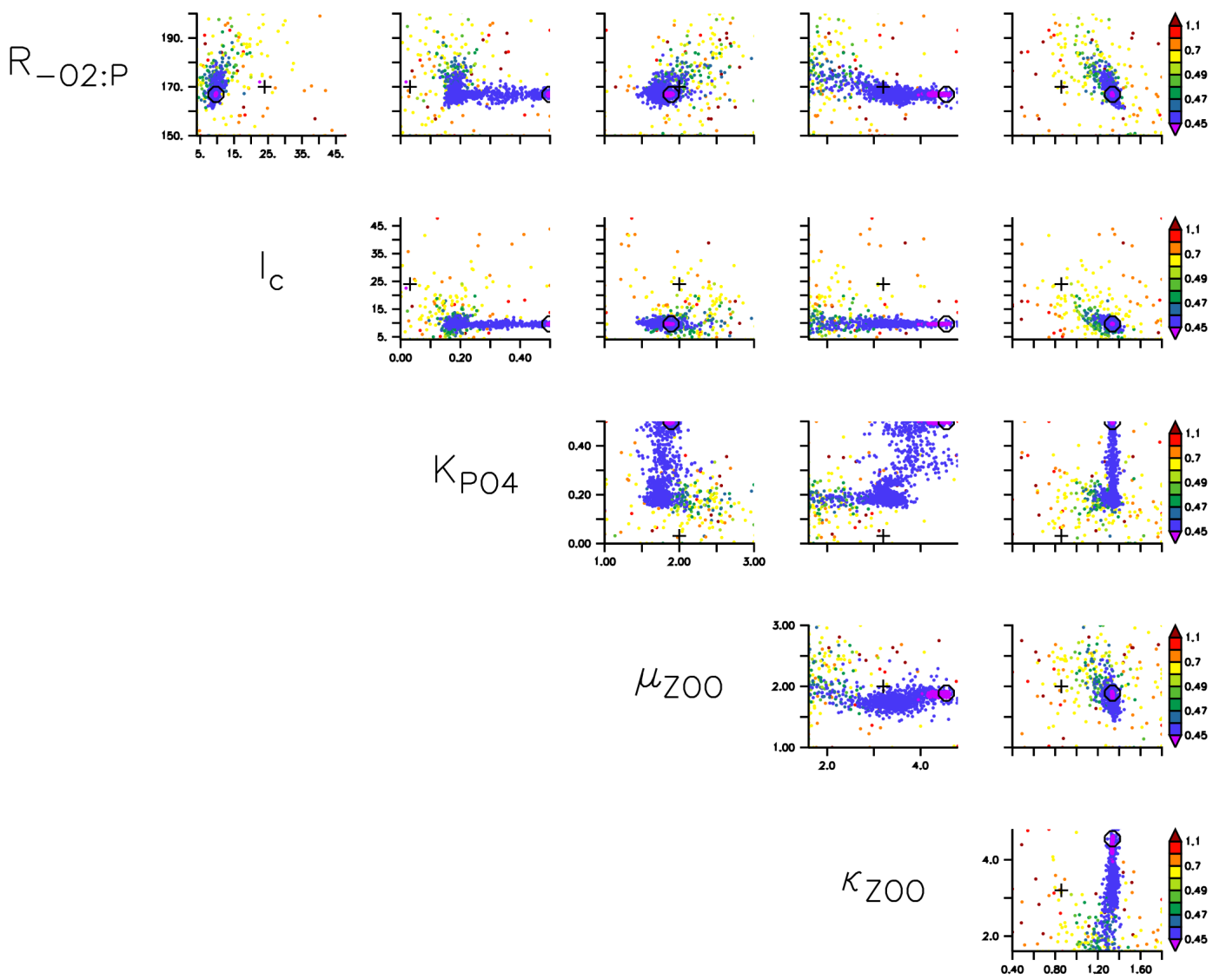

Figure 15. As Fig. 12, but for OBS-NARR.

optimizers' implementation supports asynchronous communication. An example of this approach is dealt with in Kliemann et al. (2013). There, aborting fitness calculations reduces the computational effort by orders of magnitude, since the considered combinatorial problem is of minimax type. However, short-cut fitness computation concerning ocean models requires a more elaborated method and is not expected to reach similar savings.

\subsection{Misfit function and parameter identifiability}

In our study we chose annual means of dissolved nutrients and oxygen on a rather coarse spatial grid as a measure for model skill. By doing so, we avoid problems associated with time lags (e.g., in phytoplankton blooms, which would result in time lags of nutrient depletion) or meso- and submesoscale spatial structures (see, e.g., Wallhead et al., 2006), obviously at the cost of precisely resolving parameters related to the biological system in surface layers. Possibly as a consequence of this particular misfit function, the parameters that could be fitted best are parameters that are mostly influential in determining the nutrient or oxygen distribution on large spatial and temporal scales, such as the stoichiometric ratio between oxygen and phosphorus, $R_{-\mathrm{O}_{2}}: \mathrm{P}$, or the parameter that determines particle sinking speed, $b$ (see also Kriest et al., 2012). Our model optimizations against observations so far confirm a stoichiometry of $R_{-\mathrm{O}_{2}: \mathrm{P}} \approx$ $170 \mathrm{mmol} \mathrm{O}_{2}$ : mmol $\mathrm{P}$, in agreement with observational estimates (Takahashi et al., 1985; Anderson and Sarmiento, 1994 ), but suggest an increase in $b$ towards $\approx 1.3$. The latter is to some extent in agreement with results obtained by Kwon and Primeau (2006, 2008), who found an optimal $b$ of 1 when fitting a simple global model against observed inorganic tracers. It should be kept in mind, however, that the $b$ obtained in our study not only represents particle sinking speed, but also accounts for the effect of numerical diffusion on our rather coarse vertical grid (Kriest and Oschlies, 2011). Accordingly, the "true" $b$ can be regarded as being about 10$20 \%$ smaller than obtained by our study. Also, as has been shown earlier (Kriest and Oschlies, 2013), the lower boundary condition simulated by benthic exchange can be very important for the ability of phosphate and oxygen to constrain particle sinking; therefore, the results obtained in our study 

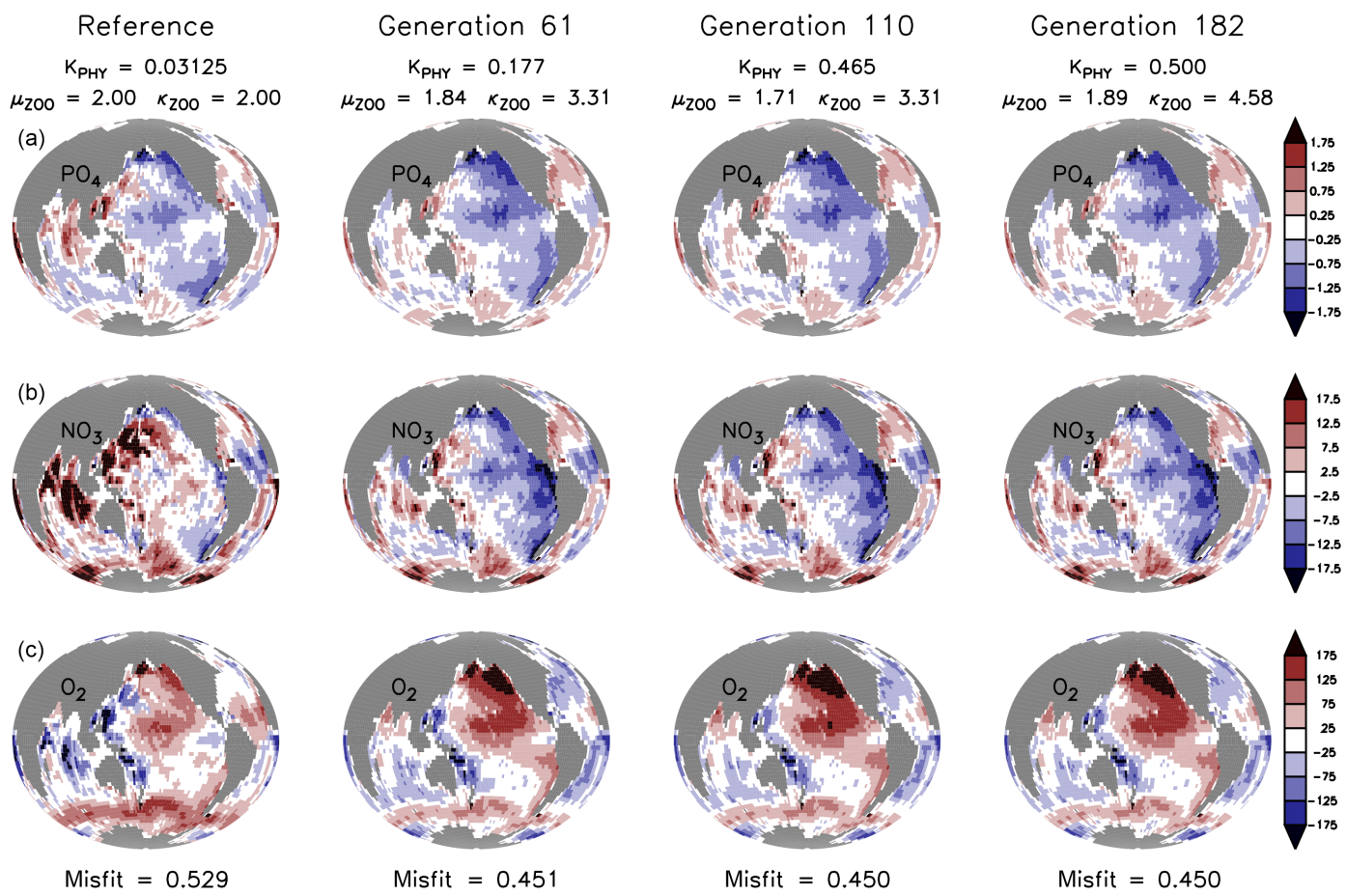

Figure 16. Model deviations from observations of vertically integrated phosphate (top), nitrate (middle), and oxygen (bottom) for the reference run, and three generations $(61,110,182)$ of OBS-NARR. See the blue lines in Fig. 14 for parameter values in this generation. For each generation, we chose the best (with respect to misfit) individual for plotting. The misfit is $0.451,0.450$, and 0.450 for generations 61 , 110 , and 182 , respectively.

should be regarded as specific to this particular biogeochemical model.

Our optimizations against observations with wide and narrow boundaries for zooplankton parameters produced two solutions with quite similar misfit, but with very different biological parameters, and consequently different fluxes and concentrations of organic components in the surface layers. Using wide boundary constraints for zooplankton parameters resulted in a solution where zooplankton is almost extinct, while phytoplankton and DOM concentration are far too high. Solutions of optimizations with unrealistic parameter values or concentrations for zooplankton have been observed earlier (Schartau et al., 2001; Ward et al., 2010), and point towards a necessity to better constrain this compartment. Increasing the population size $\lambda$ of CMA-ES in optimization OBS-WIDE-20 could cure this problem, but at the cost of a high computational demand. Restricting the range of zooplankton parameters resulted in a better fit to nutrient and oxygen; more importantly, concentrations and fluxes in the latter solution are much more realistic, confirming in the latter parameter set. This illustrates the potential benefit of a sound a priori knowledge of parameter ranges, both in terms of biogeochemical and computational performance.

Another possibility to avoid undesired effects like nearly extinct zooplankton is to introduce further criteria that take account of this issue. A technically easy approach would be to add further objective terms to the misfit function. But facing complex model interactions, it can become difficult to find suitable weights for the different terms in order to force solutions to become a desired compromise of objectives. An alternative is to deal with more than one objective function, say $f_{1}, f_{2}, \ldots, f_{k}$. For example, we can define the deviation of zooplankton mass from observed values as a second objective. Now, two solutions $x \neq y$ are said to be incomparable if $f_{i}(x)>f_{i}(y)$ but $f_{j}(x)<f_{j}(y)$ for some $i \neq j$. Multiobjective optimization algorithms aim to find (a limited number of) good incomparable solutions, from which the user can make a final choice that is a good compromise in his/her opinion. The topic of multi-objective optimization is intensively regarded with EAs (Deb, 2001) and EDAs (Hauschild and Pelikan, 2011), including CMA-ES (Igel et al., 2007).

Nevertheless, even for the more realistic optimizations OBS-WIDE-20, OBS-NARR, and OBS-NARR-R, we find similar misfits for a rather wide range of some phytoplankton and zooplankton parameters, pointing towards an indeterminacy of these parameters when using the current misfit function. While it cannot be ruled out that this arises from a correlation among these parameters, even simpler biogeochemical models with less degrees of freedom might be difficult to constrain from nutrient data alone: problems were also encountered by Kwon and Primeau (2006) when optimizing $b$, DOP production, and its decay rate against phosphate on 
a global scale. They found that phosphate data alone were not sufficient to resolve parameters associated with DOP, but several equally good fits could be obtained with different sets of parameters. It remains to be investigated whether this is related to the lack of seasonal data or to phosphate concentration being weakly dependent on dissolved or particular organic matter concentration. Subsequent studies with different misfit functions that, for example, resolve monthly changes, target the representation of surface nutrients (e.g., by using a weighted, relative misfit; Kriest et al., 2010) or add additional tracers to the misfit function (e.g., by combining chlorophyll derived from remote sensing with nitrate observations; see also Tjiputra et al., 2007) will reveal the effect of the assumptions made for the misfit function with respect to constraining these parameters.

\subsection{Future directions}

Even the use of observations more closely related to surface biology may not resolve the problem of indeterminacy, as shown by Ward et al. (2010) in optimizations of two different, zero-dimensional biogeochemical models. As in earlier zero- and three-dimensional studies (e.g., Friedrichs, 2001; Schartau et al., 2001; Kwon and Primeau, 2006, 2008), they found almost identical misfits for a wide range of parameters, an indication that these models are underdetermined, particularly when attempting to estimate more than about 10 parameters. In our study we have chosen to tune a rather moderate number of six parameters, but already noted some difficulty in constraining two of these. A potential solution could be to fix certain parameters to prior values, and thereby decrease the dimension of the parameter space to be estimated. However, as pointed out by Ward et al. (2010), this may lead to an underestimate of model uncertainty, and therefore not be the ultimate solution to this problem. Future studies will address these problems by testing different combinations of parameters, in conjunction with different misfit functions.

The above-mentioned problems may even increase if we move towards more sparsely sampled, biased, or noisy data. So far, for the twin experiment as well as for the optimization against observations, we assume perfect data coverage. However, sparse data sets (as usually available from cruises or time series stations) as well as the influence of noise have been shown to be very influential for the ability of an optimization to recover results from zero- (Friedrichs, 2001; Schartau et al., 2001; Löptien and Dietze, 2015) and threedimensional (Tjiputra et al., 2007) twin experiments. The presence of noise or measurement errors should be reflected in the termination criterion for optimization; this will, for some parameters, influence the estimates' optimum values (see Fig. 8 of Schartau et al., 2016). Future studies will have to address to what extent noise will affect the threedimensional optimizations presented here and how this parameter uncertainty will map onto model fluxes, or even transient scenarios.
While we found a decrease in the twin experiment's misfit to almost zero, the misfit of the optimization against observations remained relatively high (on average, about $15 \%$ of global mean tracer concentrations). Potential reasons for this are an inappropriate biogeochemical model structure, wrong choice of parameters to be optimized, or flaws in the physical model. For example, it is well known that coarse-resolution models do not resolve physical processes of the Equatorial Pacific current system (Dietze and Loeptien, 2013), which may result in an attempt of the optimization to "cure" deficient physics by changing biogeochemical parameters. This feature might also explain some of the sensitivities - or lack of - found by Kwon and Primeau (2006). Solutions to this potential flaw could be to exclude regions from the misfit that are known to be not well represented by the physical model, or to weigh biogeochemical misfits by the model's fit to observations of physical data.

To summarize, any global model study that aims to inversely determine parameters of a global biogeochemical ocean model in an attempt to find the model setup "best" suited for a particular application (and circulation) has to consider five tasks: (1) investigate model solutions on the appropriate (depending on tunable parameters) timescales, possibly including long, millennial simulations; (2) address the potential of local minima (depending on the topography of the misfit function); (3) investigate different parameter combinations and boundaries, including the misfit function's sensitivity to them; (4) disentangle the effects of physical and biogeochemical models on model-data misfit; and (5) investigate the effect of misfit function, including data distribution and availability in model assessment. This last point also includes decisions about weights applied to different data sets, or for a particular form of misfit function, which may be very influential for the optimal parameter choice (Evans, 2003). It also depends on the desired application of the model, and the scientific question it is supposed to address.

\section{Conclusions}

We have presented a framework for the optimization of global biogeochemical ocean models that combines an offline approach for transport of biogeochemical tracers with an estimation of distribution algorithm (Covariance Matrix Adaption Evolution Strategy, CMA-ES). A twin experiment revealed a good performance of this algorithm with respect to recovering six parameters that are associated with various timescales and space scales. Optimizations against observations of annual mean nutrients and oxygen could reduce the misfit of the model to some extent; however, even for the "best" model solution the remaining misfit is still $\approx 15 \%$ of global mean tracer concentrations, which might be related to inappropriate physics. Tests with a different circulation (which is easy to exchange with the current framework) will provide more insight into the impact of physical forcing on 
the ability of the biogeochemical model to fit the observations.

Encouragingly, parameter sets associated with the lowest misfit to dissolved inorganic tracers also show the best fit to global mean tracer fluxes not considered during optimization. This increases our confidence in the method presented here. Some parameter estimates are associated with a rather high level of uncertainty. Incorporating different or additional data sets that more closely relate to the parameters to be optimized can help to improve estimates for these parameters. Likewise, observations that provide information about the upper and lower bounds of biological parameters - such as zooplankton grazing and mortality rates - will provide a good guidance for future optimization studies and lower their computational demand.

\section{Code availability}

The source code of MOPS coupled to TMM, as well as the optimization framework, are available as the Supplement. The most recent TMM source code, forcing, etc., are available at https://github.com/samarkhatiwala/tmm. 


\section{Appendix A: Source code}

As research questions may diverge strongly (and therefore, also the different user groups, hardware, biogeochemical models, and circulations), we aimed to construct a tool that is as generic and universally applicable as possible, with a high level of portability among different architectures. The model-optimization framework of TMM comprises new subroutines for data assimilation and misfit function evaluation, as well as monitor routines to facilitate run-time checks of model state, and a more generic coupling interface for biogeochemistry. It can thus easily be applied within an optimization framework. While we here focus on the coarseresolution model, we note that the generic structure of the TMM framework allows the user to easily switch between transport matrices, once these are available. Likewise, coupling different biogeochemical models to the framework only requires editing of a (few) interface subroutines. Finally, in principle it should be possible to exchange the optimization algorithm by any other algorithm that requires only model misfit as input and provides a set of parameter files as output.

\section{A1 MOPS-2.0 biogeochemical subroutines}

Besides the stand-alone, forward integration of a global biogeochemical model, two additional tasks are required for optimization: computation and output of misfit, and input of trial sets of parameters passed to the model by the optimizer. In the following, files relevant for input of parameter vectors and computation of misfit that have been added or changed (with respect to MOPS-1.0; see Kriest and Oschlies, 2015) are shown in boldface. An overview of the model structure and layout, with emphasis on those parts that affect computation of biogeochemical fluxes and tracers, optimization, and parameter handling, is given in the Supplement.

As noted in Kriest and Oschlies (2015), the code consists of two files with outer routines that connect to the main driver code tmm_main. c, and inner routines that contain the local biogeochemical sources and sinks, and define the biogeochemical parameters. These routines communicate via common blocks in header files.

1. external_forcing_mops_biogeochem.c is the first interface between MOPS and the TMM. Besides input and output of files and runtime parameters, it determines from runtime options whether a parameter file should be read, as well as its name. It assembles model equivalents for the misfit function and passes it to the main driver code, tmm_main. c. It calls the following subroutines.

1.2. mops_biogeochem_ini.F: interface between (1) and (1.2.1). It calls the following.

1.2.1. BGC_INI.F assigns biogeochemical parameters. The routine distinguishes between parameters that stay fixed and derived parameters that depend on parameters read during runtime. For example, the stoichiometric ratio $\mathrm{O}_{2}: \mathrm{P}$ determines the stoichiometry for nitrate loss during denitrification (Paulmier et al., 2009). Thus, if the former changes, the latter will have to be recalculated. The routine is called every time after a new parameter vector has been read.

1.3. mops_biogeochem_set_params.F maps the vector of parameters read by (1) to symbolic names used by MOPS. Each call to (1.3) is followed by a call to (1.2) and (1.2.1), to recalculate dependent parameters.

1.1. mops_biogeochem_copy_data.F: interface between (1) and (1.2) and (1.4).

1.4. mops_biogeochem_model.F: interface between (1) and (1.4.1). It calls

1.4.1. BGC_MODEL.F: calculates biogeochemical sources and sinks. It now also assigns state variables to arrays that will be passed to the misfit function.

1.5. mops_biogeochem_diagnostics.F: interface (for diagnostic output) between (1) and (1.4.1).

1.6. mops_biogeochem_misfit.F: interface for misfit computation between (1) and (1.4.1).

2. tmm_misfit.c initializes and carries out misfit computation and writes misfit to either binary or ASCII files. It communicates with the biogeochemical model in (1) via (b).

Communication between the different modules is carried out mainly via several header files:

a. mops_biogeochem.h introduces subroutines to (1);

b. mops_biogeochem_misfit_data.h passes information related to misfit computation between (1) and (2).

c. BGC_PARAMS.h passes biogeochemical parameters and profiles of tracers between all different modules called by (1).

d. BGC_DIAGNOSTICS.h passes diagnostic variables from (1.4.1) to (1.5).

e. BGC_MISFIT . h passes misfit variables from (1.4.1) to (1.6).

f. BGC_CONTROL.h passes the time step and geometry between (1.2) and (1.2.1), (1.4), and (1.4.1);

g. tmm_external_forcing.h introduces subroutines in (1) to tmm_main.c; and

h. tmm_misfit.h introduces subroutines in (2) to tmm_main.c. 
Finally, one may want to prevent computation of a simulation if during spinup some parameter values or concentrations lead to erroneous (e.g., negative) tracer concentrations. Routine tmm_monitor.c may serve as a module to monitor state variables, or other model properties (not used in the current setup presented here).

\section{A2 Optimization}

As noted above, the framework presented here is set up such that a serial script serial. job calls the optimization routine (in our case CMA-ES), which computes a population of size $=\lambda$ of parameter vectors, stored in ASCII files. The same script then calls a parallel script parallel.job, which starts $\lambda$ model simulations. During these simulations, the parameter files are read, and a spinup is carried out for each individual setup. The individual model runs then output the misfit function to specified files. When all jobs are finished, script parallel. job invokes script serial. job again, etc. Thus, communication between both alternating steps (creation of parameter vectors and computation of the resulting misfit function) is carried out by these parameter and misfit files. In addition, file nIter.txt keeps track of the progress of optimization, and provides the information on which generation is to be computed; it also contains the runtime parameters for the optimizer, CMA-ES. See the information in the Supplement for more details on how this setup works, and how to specify biogeochemical and optimizer parameters used, e.g., in the work presented here. 


\section{The Supplement related to this article is available online at doi:10.5194/gmd-10-127-2017-supplement.}

Acknowledgements. This work is a contribution to DFG-supported project SFB754 and to the research platforms of DFG cluster of excellence The Future Ocean. We thank Nikolaus Hansen for support on and open access to the CMA-ES code. Parallel supercomputing resources have been provided by the NorthGerman Supercomputing Alliance (HLRN). The authors wish to acknowledge use of the Ferret program of the NOAA's Pacific Marine Environmental Laboratory for analysis and graphics in this paper. We thank Momme Butenschön and an anonymous reviewer for their very constructive and helpful comments.

Edited by: C. Sierra

Reviewed by: M. Butenschön and one anonymous referee

\section{References}

Anderson, L.: On the hydrogen and oxygen content of marine phytoplankton, Deep-Sea Res. Pt. I, 42, 1675-1680, 1995.

Anderson, L. and Sarmiento, J.: Redfield ratios of remineralization determined by nutrient data analysis, Global Biogeochem. Cy., 8, 65-80, 1994.

Arnold, D. V.: Weighted multirecombination evolution strategies, Lect. Notes Comput. Sc., 361, 18-37, 2006.

Athias, V., Mazzega, P., and Jeandel, C.: Selecting a global optimization method to estimate the oceanic particle cycling rate constants, J. Mar. Res., 58, 675-707, 2000.

Auger, A., Brockhoff, D., and Hansen, N.: Benchmarking the local metamodel CMA-ES on the noiseless BBOB'2013 test bed, in: Genetic and Evolutionary Computation Conference, GECCO 2013, Amsterdam, the Netherlands, 6-10 July 2013, Companion Material Proceedings, 1225-1232, 2013.

Babu, G. S. S., Das, D. B., and Patvardhan, C.: Solution of realparameter optimization problems using novel Quantum Evolutionary Algorithm with applications in power dispatch, in: Proceedings of the IEEE Congress on Evolutionary Computation, CEC 2009, Trondheim, Norway, 18-21 May, 2009, 1920-1927, 2009.

Beyer, H.-G.: The theory of evolution strategies, Springer, Berlin, 2001.

Boulahdid, M. and Minster, J.-F.: Oxygen consumption and nutrient regeneration ratios along isopycnal horizons in the Pacific Ocean, Mar. Chem., 26, 133-153, 1989.

Buesseler, K., Lamborg, C., Boyd, P., Lam, P., Trull, T., Bidigare, R., Bishop, J., Casciotti, K., Dehairs, F., Elskens, M., Honda, M., Karl, D., Siegel, D., Silver, M., Steinberg, D., Valdes, J., Mooy, B. V., and Wilson, S.: Revisiting carbon flux through the ocean's twilight zone, Science, 316, 567-570, 2007.

Cabré, A., Marinov, I., Bernardello, R., and Bianchi, D.: Oxygen minimum zones in the tropical Pacific across CMIP5 models: mean state differences and climate change trends, Biogeosciences, 12, 5429-5454, doi:10.5194/bg-12-5429-2015, 2015.
Carr, M.-E., Friedrichs, M., Schmeltz, M., Aitac, M., Antoine, D., Arrigo, K., Asanuma, I., Aumont, O., Barber, R., Behrenfeld, M., Bidigare, R., Buitenhuis, E., Campbell, J., Ciotti, A., Dierssen, H., Dowell, M., Dunne, J., Esaias, W., Gentili, B., Gregg, W., Groom, S., Hoepffner, N., Ishizaka, J., Kameda, T., Quere, C. L., Lohrenz, S., Marra, J., Lino, F. M., Moore, K., Morel, A., Reddy, T., Ryan, J., Scardi, M., Smyth, T., Turpie, K., Tilstone, G., Waters, K., and Yamanaka, Y.: A comparison of global estimates of marine primary production from ocean color, Deep-Sea Res. Pt. II, 53, 741-770, doi:10.1016/j.dsr2.2006.01.028, 2006.

Collos, Y., Vaquer, A., and Souchou, P.: Acclimation of nitrate uptake by phytoplankton to high substrate levels, J. Phycol., 41, 466-478, 2005.

Cover, T. M. and Thomas, J. A.: Elements of Information Theory, John Wiley \& Sons, Hoboken, NJ, 2006.

Deb, K.: Multi-objective optimization using evolutionary algorithms, John Wiley \& Sons, Hoboken, NJ, 2001.

Dietze, H. and Loeptien, U.: Revisiting "nutrient trapping” in global coupled biogeochemical ocean circulation models, Global Biogeochem. Cy., 27, 265-284, doi:10.1002/gbc.20029, 2013.

Dunne, J. P., Sarmiento, J. L., and Gnanadesikan, A.: A synthesis of global particle export from the surface ocean and cycling through the ocean interior and on the seafloor, Global Biogeochem. Cy., 21, GB4006, doi:10.1029/2006GB002907, 2007.

Evans, G. T.: Defining misfit between biogeochemical models and data sets, J. Mar. Syst., 40-41, 49-54, 2003.

Fasham, M. and Evans, G.: The use of optimization techniques to model marine ecosystem dynamics at the JGOFS Station at $47^{\circ}$ N, $20^{\circ}$ W, Philos. T. Roy. Soc. B, 348, 203-209, 1995.

Friedrichs, M. A. M.: A data assimilative marine ecosystem model of the central equatorial Pacific: Numerical twin experiments, Jour. Mar. Res., 59, 859-894, 2001.

Garcia, H. E., Locarnini, R. A., Boyer, T. P., and Antonov, J. I.: World Ocean Atlas 2005, Vol. 4: Nutrients (phosphate, nitrate, silicate), in: NOAA Atlas NESDIS 64, edited by: Levitus, S., US Government Printing Office, Washington, DC, available at: http://iridl.ldeo.columbia.edu/SOURCES/.NOAA/ .NODC/.WOA05/, 2006a.

Garcia, H. E., Locarnini, R. A., Boyer, T. P., and Antonov, J. I.: World Ocean Atlas 2005, Vol. 3: Dissolved Oxygen, Apparent Oxygen Utilization, and Oxygen Saturation, in: NOAA Atlas NESDIS 63, edited by: Levitus, S., US Government Printing Office, Washington, DC, available at: http://iridl.ldeo.columbia. edu/SOURCES/.NOAA/.NODC/.WOA05/, $2006 \mathrm{~b}$.

Hansen, N.: The CMA evolution strategy: a comparing review, in: Towards a new evolutionary computation, Advances on estimation of distribution algorithms, edited by: Lozano, J. A., Larranaga, P., Inza, I., and Bengoetxea, E., Springer, 75-102, 2006.

Hansen, N.: The CMA Evolution Strategy: A Tutorial, arXiv:1604.00772v1 [cs.LG], 2016.

Hansen, N. and Kern, S.: Evaluating the CMA Evolution Strategy on Multimodal Test Functions, in: Parallel Problem Solving from Nature PPSN VIII, edited by: Yao, X., Burke, E. K., Lozano, J. A., Smith, J., Merelo Guervós, J. J., Bullinaria, J. A., Rowe, J. E., Tiño, P., Kabán, A., and Schwefel, H.-P., Lecture Notes in Computer Science, Springer, 3242, 282-291, 2004.

Hansen, N. and Ostermeier, A.: Completely Derandomized SelfAdaptation in Evolution Strategies, Evol. Comput., 9, 159-195, 2001. 
Hansen, N., Finck, S., Ros, R., and Auger, A.: Real-Parameter Black-Box Optimization Benchmarking 2009: Noiseless Function Definitions, Tech. Rep. inria-00362633, INRIA, Le Chesnay, France, available at: https://hal.inria.fr/inria-00362633/ document, 2009a.

Hansen, N., Niederberger, A. S. P., Guzzella, L., and Koumoutsakos, P.: A Method for Handling Uncertainty in Evolutionary Optimization with an Application to Feedback Control of Combustion, IEEE T. Evolut. Comput., 13, 180-197, 2009 b.

Hansen, N., Auger, A., Ros, R., Finck, S., and Posík, P.: Comparing results of 31 algorithms from the black-box optimization benchmarking BBOB-2009, in: Proceedings of the Genetic and Evolutionary Computation Conference, GECCO 2010, Portland, Oregon, USA, 7-11 July 2010, 1689-1696, 2010.

Hansen, N., Arnold, D. V., and Auger, A.: Evolution Strategies, in: Springer Handbook of Computational Intelligence, Springer, Berlin, 871-898, 2015.

Hauschild, M. and Pelikan, M.: An Introduction and Survey of Estimation of Distribution Algorithms, available at: http://medal-lab. org/files/2011004_rev1.pdf, 2011.

Hemmings, J. C. P., Challenor, P. G., and Yool, A.: Mechanistic site-based emulation of a global ocean biogeochemical model (MEDUSA 1.0) for parametric analysis and calibration: an application of the Marine Model Optimization Testbed (MarMOT 1.1), Geosci. Model Dev., 8, 697-731, doi:10.5194/gmd-8-6972015, 2015.

Henson, S. A., Sanders, R., and Madsen, E.: Global patterns in efficiency of particulate organic carbon export and transfer to the deep ocean, Global Biogeochem. Cy., 26, GB1028, doi:10.1029/2011GB004099, 2012.

Honjo, S., Manganini, S. J., Krishfield, R. A., and Francois, R.: Particulate organic carbon fluxes to the ocean interior and factors controlling the biological pump: A synthesis of global sediment trap programs since 1983, Prog. Oceanogr., 76, 217-285, doi:10.1016/j.pocean.2007.11.003, 2008.

Igel, C., Hansen, N., and Roth, S.: Covariance Matrix Adaptation for Multi-objective Optimization, Evol. Comput., 15, 1-28, 2007

Jin, Y.: Surrogate-assisted evolutionary computation: Recent advances and future challenges, Swarm Evol. Comput., 1, 61-70, 2011

Jost, L.: Entropy and Diversity, Oikos, 113, 363-375, 2006.

Kern, S., Hansen, N., and Koumoutsakos, P.: Local Meta-models for Optimization Using Evolution Strategies, in: Parallel Problem Solving from Nature - PPSN IX, 9th International Conference, Reykjavik, Iceland, 9-13 September 2006, 939-948, 2006.

Khatiwala, S.: A computational framework for simulation of biogeochemical tracers in the ocean, Global Biogeochem. Cy., 21, GB3001, doi:10.1029/2007GB002923, 2007.

Kliemann, L., Kliemann, O., Patvardhan, C., Sauerland, V., and Srivastav, A.: A New QEA Computing Near-Optimal LowDiscrepancy Colorings in the Hypergraph of Arithmetic Progressions, in: Proceedings of the 12th International Symposium on Experimental and Efficient Algorithms, edited by: Bonifaci, V., Demetrescu, C., and Marchetti-Spaccamela, A., Lecture Notes in Computer Science, 7933, 67-78, 2013.

Kriest, I. and Oschlies, A.: On the treatment of particulate organic matter sinking in large-scale models of marine biogeochemi- cal cycles, Biogeosciences, 5, 55-72, doi:10.5194/bg-5-55-2008, 2008.

Kriest, I. and Oschlies, A.: Numerical effects on organic matter sedimentation and remineralization in biogeochemical ocean models, Ocean Modell., 39, 275-283, doi:10.1016/j.ocemod.2011.05.001, 2011.

Kriest, I. and Oschlies, A.: Swept under the carpet: organic matter burial decreases global ocean biogeochemical model sensitivity to remineralization length scale, Biogeosciences, 10, 8401-8422, doi:10.5194/bg-10-8401-2013, 2013

Kriest, I. and Oschlies, A.: MOPS-1.0: towards a model for the regulation of the global oceanic nitrogen budget by marine biogeochemical processes, Geosci. Model Dev., 8, 2929-2957, doi:10.5194/gmd-8-2929-2015, 2015.

Kriest, I., Khatiwala, S., and Oschlies, A.: Towards an assessment of simple global marine biogeochemical models of different complexity, Prog. Oceanogr., 86, 337-360, doi:10.1016/j.pocean.2010.05.002, 2010.

Kriest, I., Oschlies, A., and Khatiwala, S.: Sensitivity analysis of simple global marine biogeochemical models, Global Biogeochem. Cy., 26, GB2029, doi:10.1029/2011GB004072, 2012.

Kwon, E. Y. and Primeau, F.: Optimization and sensitivity study of a biogeochemistry ocean model using an implicit solver and in situ phosphate data, Global Biogeochem. Cy., 20, GB4009, doi:10.1029/2005GB002631, 2006.

Kwon, E. Y. and Primeau, F.: Optimization and sensitivity of a global biogeochemistry ocean model using combined in situ DIC, alkalinity, and phosphate data, J. Geophys. Res., 113, C0801, doi:10.1029/2007JC004520, 2008.

Letscher, R. T., Moore, J. K., Teng, Y.-C., and Primeau, F.: Variable $\mathrm{C}: \mathrm{N}: \mathrm{P}$ stoichiometry of dissolved organic matter cycling in the Community Earth System Model, Biogeosciences, 12, 209-221, doi:10.5194/bg-12-209-2015, 2015.

Löptien, U. and Dietze, H.: Constraining parameters in marine pelagic ecosystem models - is it actually feasible with typical observations of standing stocks?, Ocean Sci., 11, 573-590, doi:10.5194/os-11-573-2015, 2015.

Loshchilov, I., Schoenauer, M., and Sebag, M.: Self-adaptive surrogate-assisted covariance matrix adaptation evolution strategy, Genetic and Evolutionary Computation Conference, GECCO 2012, Philadelphia, PA, USA, 7-11 July 2012, 321328, 2012.

Lutz, M., Caldeira, K., Dunbar, R., and Behrenfeld, M. J.: Seasonal rhythms of net primary production and particulate organic carbon flux to depth describe biological pump efficiency in the global ocean, J. Geophys. Res., 113, C10011, doi:10.1029/2007JC003706, 2007.

Marsay, C. M., Sanders, R., Henson, S., Pabortsava, K., Achterberg, E., and Lampitt, R.: Attenuation of sinking particulate organic carbon flux through the mesopelagic ocean, P. Natl. Acad. Sci. USA, 112, 1089-1094, 2015.

Marshall, J., Adcroft, A., Hill, C., Perelman, L., and Heisey, C.: A finite-volume, incompressible Navier-Stokes model for studies of the ocean on parallel computers, J. Geophys. Res., 102, 57335752, 1997.

Martin, J. H., Knauer, G. A., Karl, D. M., and Broenkow, W. W.: VERTEX: carbon cycling in the Northeast Pacific, Deep-Sea Res., 34, 267-285, 1987. 
Najjar, R. G., Jin, X., Louanchi, F., Aumont, O., Caldeira, K., Doney, S. C., Dutay, J.-C., Follows, M., Gruber, N., Joos, F., Lindsay, K., Maier-Reimer, E., Matear, R., Matsumoto, K., Monfray, P., Mouchet, A., Orr, J. C., Plattner, G.-K., Sarmiento, J. L., Schlitzer, R., Slater, R. D., Weirig, M.-F., Yamanaka, Y., and Yool, A.: Impact of circulation on export production, dissolved organic matter and dissolved oxygen in the ocean: Results from Phase II of the Ocean Carbon-cycle Model Intercomparison Project (OCMIP-2), Global Biogeochem. Cy., 21, GB3007, doi:10.1029/2006GB002857, 2007.

Orr, J., Maier-Reimer, E., Mikolajewicz, U., Monfray, P., Sarmiento, J., Toggweiler, J., Taylor, N., Palmer, J., Gruber, N., Sabine, C., Le Quere, C., Key, R., and Boutin, J.: Estimates of anthropogenic carbon uptake from four three-dimensional global ocean models, Global Biogeochem. Cy., 15, 43-60, doi:10.1029/2000GB001273, 2001.

Patvardhan, C., Bansal, S., and Srivastav, A.: Quantum-Inspired Evolutionary Algorithm for difficult knapsack problems, Memetic Computing, 7, 135-155, 2015.

Patvardhan, C., Bansal, S., and Srivastav, A.: Parallel improved quantum inspired evolutionary algorithm to solve large size Quadratic Knapsack Problems, Swarm Evol. Comput., 26, 175190, 2016.

Paulmier, A., Kriest, I., and Oschlies, A.: Stoichiometries of remineralisation and denitrification in global biogeochemical ocean models, Biogeosciences, 6, 923-935, doi:10.5194/bg-6923-2009, 2009.

Priess, M., Koziel, S., and Slawig, T.: Marine ecosystem model calibration with real data using enhanced surrogatebased optimization, J. Computational Science, 4, 423-437, doi:10.1016/j.jocs.2013.04.001, 2013.

Rückelt, J., Sauerland, V., Slawig, T., Srivastav, A., Ward, B., and Patvardhan, C.: Parameter Optimization and Uncertainty Analysis in a Model of Oceanic $\mathrm{CO}_{2}$ Uptake Using a Hybrid Algorithm and Algorithmic Differentiation, Nonlinear Anal.-Real., 11, 3993-4009, 2010.

Schartau, M. and Oschlies, A.: Simultaneous data-based optimization of a 1D-ecosystem model at three locations in the North Atlantic: Part II - Standing stocks and nitrogen fluxes, J. Mar. Res., 61, 795-821, 2003a.

Schartau, M. and Oschlies, A.: Simultaneous data-based optimization of a 1D-ecosystem model at three locations in the North Atlantic: Part I - Method and parameter estimates, J. Mar. Res., 61, 765-793, 2003b.
Schartau, M., Oschlies, A., and Willebrand, J.: Parameter estimates of a zero-dimensional ecosystem model applying the adjoint method, Deep-Sea Res. Pt. II, 48, 1796-1800, 2001.

Schartau, M., Wallhead, P., Hemmings, J., Löptien, U., Kriest, I., Krishna, S., Ward, B. A., Slawig, T., and Oschlies, A.: Reviews and syntheses: Parameter identification in marine planktonic ecosystem modelling, Biogeosciences Discuss., doi:10.5194/bg2016-242, in review, 2016.

Schmoker, C., Hernandez-Leon, S., and Calbet, A.: Microzooplankton grazing in the oceans: impacts, data variability, knowledge gaps and future directions, Jour. Plank. Res., 35, 691-706, doi:10.1093/plankt/fbt023, 2013.

Séférian, R., Gehlen, M., Bopp, L., Resplandy, L., Orr, J. C., Marti, O., Dunne, J. P., Christian, J. R., Doney, S. C., Ilyina, T., Lindsay, K., Halloran, P. R., Heinze, C., Segschneider, J., Tjiputra, J., Aumont, O., and Romanou, A.: Inconsistent strategies to spin up models in CMIP5: implications for ocean biogeochemical model performance assessment, Geosci. Model Dev., 9, 18271851, doi:10.5194/gmd-9-1827-2016, 2016.

Takahashi, T., Broecker, W., and Langer, S.: Redfield ratio based on chemical data from isopycnal surfaces, J. Geophys. Res., 90, 6907-6924, 1985.

Tjiputra, J. F., Polzin, D., and Winguth, A.: Assimilation of seasonal chlorophyll and nutrient data into an adjoint three-dimensional ocean carbon cycle model: Sensitivity analysis and ecosystem parameter optimization, Glob. Biogeochem. Cy., 21, GB1001, doi:10.1029/2006GB002745, 2007.

Van Mooy, B., Keil, R., and Devol, A.: Impact of suboxia on sinking particulate organic carbon: Enhanced carbon flux and preferential degradation of amino acids via denitrificiation, Geochim. Cosmochim. Ac., 66, 457-465, f5, 2002.

Wallhead, P., Martin, A., Srokosz, M., and Fasham, M.: Accounting for unresolved spatial variability in marine ecosystems using time lags, Jour. Mar. Res., 64, 881-914, 2006.

Wallmann, K.: Phosphorus imbalance in the global ocean?, Global Biogeochem. Cy., 24, GB4030, doi:10.1029/2009GB003643, 2010.

Ward, B.: Marine Ecosystem Model Analysis Using Data Assimilation, $\mathrm{PhD}$ thesis, Univ. Southampton, School of Ocean and Earth Science, 2009.

Ward, B., Friedrichs, M. A. M., Anderson, T., and Oschlies, A.: Parameter optimisation techniques and the problem of underdetermination in marine biogeochemical models, J. Mar. Systems, 81, 34-43, doi:10.1016/j.jmarsys.2009.12.005, 2010. 